\title{
Fast difference schemes for solving high-dimensional time-fractional subdiffusion equations
}

\author{
Fanhai Zeng ${ }^{\dagger}$, Zhongqiang Zhang ${ }^{\ddagger}$, George Em Karniadakis ${ }^{\dagger, *}$
}

\begin{abstract}
In this paper, we focus on fast solvers with linearithmic complexity in space for highdimensional time-fractional subdiffusion equations. Firstly, we present two alternating direction implicit (ADI) finite difference schemes for the two-dimensional time-fractional subdiffusion equation that are convergent of order $(1+\beta)$ in time, where $\beta(0<\beta<1)$ is the fractional order. Secondly, we develop two finite difference schemes which admit fast solvers without applying ADI techniques for two-dimensional time-fractional subdiffusion. Lastly, we extend these fast solvers to three-dimensional time-fractional subdiffusion. All the non-ADI difference methods are unconditionally stable and convergent with order two in time and two or four in space. We also present several numerical experiments to verify the theoretical results.
\end{abstract}

Keywords:

Subdiffusion, fractional linear multistep method, ADI, fast Poisson solver, compact finite difference, unconditional stability, convergence.

\section{Introduction}

Anomalous diffusion, either subdiffusion or superdiffusion, is encountered in many diverse applications in science and engineering, see e.g. [1]. It is typically modeled through timefractional derivatives, which give rise to great computational complexity caused by the nonlocal nature of the fractional operators. Numerical solution of the corresponding fractional differential equations (FDEs) is particularly problematic in high dimensions, so the majority of published works deals with one-dimensional problems whereas high dimensions are usually split following a classical alternating direction implicit (ADI) method. In this paper, we consider fast finite difference methods (FDMs) with linearithmic complexity for the following

\footnotetext{
${ }^{\dagger}$ Division of Applied Mathematics, Brown University, Providence RI, 02912 (fanhai_zeng@brown.edu, george_karniadakis@brown.edu).

${ }_{\ddagger}^{\ddagger}$ Department of Mathematical Sciences, Worcester Polytechnic Institute, Worcester, MA, 01609 (zzhang7@wpi.edu).

${ }^{*}$ Corresponding author.
} 
two-dimensional time-fractional subdiffusion equation, see e.g. [1, 2, 3]:

$$
\left\{\begin{array}{l}
{ }_{C} D_{0, t}^{\beta} U=\mu \Delta U+f(x, y, t), \quad(x, y, t) \in \Omega \times(0, T], T>0, \\
U(x, y, 0)=\phi_{0}(x, y), \quad x \in \Omega \\
U(x, y, t)=0, \quad(x, y, t) \in \partial \Omega \times(0, T]
\end{array}\right.
$$

and its three-dimensional counterpart, where $\Delta=\partial_{x}^{2}+\partial_{y}^{2}, 0<\beta<1, \mu>0, \Omega=$ $\left(x_{L}, x_{R}\right) \times\left(y_{L}, y_{R}\right)$, and ${ }_{C} D_{0, t}^{\beta}$ is the $\beta$ th-order Caputo derivative operator defined by [4]

$$
{ }_{C} D_{0, t}^{\beta} U(\cdot, t)=D_{0, t}^{-(1-\beta)}\left[\partial_{t} U(\cdot, t)\right]=\frac{1}{\Gamma(1-\beta)} \int_{0}^{t}(t-s)^{-\beta} \partial_{s} U(\cdot, s) \mathrm{d} s,
$$

in which $D_{0, t}^{-\beta}$ is the fractional integral operator defined by

$$
D_{0, t}^{-\beta} U(\cdot, t)={ }_{R L} D_{0, t}^{-\beta} U(\cdot, t)=\frac{1}{\Gamma(\beta)} \int_{0}^{t}(t-s)^{\beta-1} U(\cdot, s) \mathrm{d} s, \quad \beta>0 .
$$

Many numerical methods have been proposed to solve high-dimensional fractional partial differential equations (FPDEs) like (1): see e.g. $[5,6,7,8,9]$ for space-fractional partial differential equations (PDEs), e.g. [3, 10, 11, 12, 13, 14, 15, 16, 17, 18, 19, 20, 21, 22, 23, 24] for time-fractional PDEs, and e.g. [25, 26, 27, 28, 29] for time-space-fractional diffusion; see also the recent book [30] on a review of numerical methods for FDEs. Among all the numerical methods for high-dimensional FPDEs, only the ADI method is computationally efficient to be applied to solve the resulting linear systems with linear complexity, see e.g. $[5,6,7,8,9,31,32,33,34,35]$. However, there is a noticeable difference when ADI techniques are applied to time-fractional PDEs and integer-order PDEs: the convergence rate in time is degraded by the fractional order $\beta$, see e.g. $[2,3,28,36,37,38]$, while for the integerorder PDEs, ADI techniques do not have such a limitation, see e.g. [39, 40, 41]. For ADI methods of the high-dimensional time-fractional subdiffusion equation of the type (1), the convergence rate in time is of order

- $\min \{q, 1+\beta\}$ (e.g., $q=2-\beta$ in $[2,3])$ or

- $\min \{q, 2 \beta\}$ (e.g., $q=1$ in [37], $q=2-\beta$ in [36, 3], and $q=2-\beta / 2$ in [38]), or

- $\min \{q, \beta\}$ (e.g. $q=2-\beta$ in [28]),

where $q$ is the convergence rate of the time discretization methods applied together with the ADI method. Hence, when $\beta$ is small, we achieve unsatisfactory accuracy in the existing ADI methods.

Two approaches have been proposed to improve the convergence rate of ADI methods. The first is to appropriately add some higher-order perturbation terms, see e.g. [2, 40], while the other is to use the extrapolation method, see e.g. [31, 42, 43]. For these two approaches, no theoretical analysis has been presented to guarantee the stability. 
In this paper, we use the first approach to increase the convergence rate of ADI methods, and we present two different ADI FDMs for (1). These two schemes are unconditionally stable and convergent with order $(1+\beta)$ in time and order two in space. However, the added perturbation terms may ruin the total accuracy, especially when $\beta$ is small and/or $\partial_{x}^{2} \partial_{y}^{2} U(x, y, t)$ is large, see, e.g. [40] and example 5.1 of Section 5.

We are then motivated to propose some non-ADI FDMs for the high-dimensional timefractional subdiffusion equations (1) and (41) while we can still solve them with a low computational cost that is linearithmic with respect to the number of the grid points used in FDMs. Specifically, we present two fully non-ADI difference methods for (1) using the fractional linear multistep methods developed in [44] in time discretization and the standard central difference in physical space. Thanks to the special structure of the derived coefficient matrices, we can employ a fast eigen-solver with linearithmic complexity to solve the resulting linear systems. The fast solver allows us to solve the linear system directly with $O\left(N^{2} \log (N)\right)$ operations in space when we take $N$ grid points in both $x$ and $y$ directions, instead of $O\left(N^{3}\right)$ operations for the direct solvers. We also prove that these two difference schemes are unconditionally stable with second-order accuracy both in time and space. In addition, we discuss how to achieve high-order convergence in physical space using compact finite difference schemes while we can still employ fast solvers without the ADI technique, see Section 3.2. Two compact non-ADI finite difference methods for (1) are proved to be both unconditionally stable and convergent with order two in time and four in space in Appendix.

In Section 4, we show how the methodology presented in Section 3 can be extended to solve the three-dimensional time-fractional subdiffusion equation (41) with computational cost $O\left(N^{3} \log (N)\right)$ in physical space. The present methods are expected to work for $d$-dimensional time-fractional anomalous diffusion equations with $O\left(N^{d} \log (N)\right)$ computational cost in physical space. There exist some fast solvers to solve FPDEs, such as $[9,46]$, in which the ADI technique is used to convert the high-dimensional space-fractional PDEs into a series of one-dimensional ones, then the fast solver is applied. Here, we directly use the fast solver to solve the high-dimensional problems without using the ADI technique.

The rest of this paper is as follows. In Section 2, we derive two ADI FDMs for (1) and prove their stability and convergence rate. In Section 3, we develop two FDMs for (1) and employ a fast solver to solve the resulting linear system. We also consider two compact FDMs for (1). We present the stability and convergence rates of these schemes and leave the proofs the stability and convergence in Appendix. We investigate the extension of the methodology to three-dimensional time-fractional subdiffusion in Section 4. In Section 5, we present numerical experiments to verify the theoretical results. We also present numerical comparisons between the present methods and the existing ones, both the ADI and non-ADI methods. In Section 6, we conclude and discuss our results.

\section{ADI finite difference methods}

Before presenting our numerical schemes, we introduce some notations. Let $\tau$ be the time step size and $n_{T}$ be a positive integer with $\tau=T / n_{T}$ and $t_{n}=n \tau$ for $n=0,1, \ldots, n_{T}$. 
Denote by $U(t)=U(\cdot, t)$ and $U^{n}=U^{n}(\cdot)=U\left(\cdot, t_{n}\right)$. Denote $\Delta x$ and $\Delta y$ as the step sizes in $x$ and $y$ directions, respectively, where $\Delta x=\left(x_{R}-x_{L}\right) / N_{1}$ and $\Delta y=\left(y_{R}-y_{L}\right) / N_{2}$, $N_{1}$ and $N_{2}$ are positive integers. The grid points $\left(x_{i}, y_{j}\right)$ in space are then defined as $x_{i}=x_{L}+i \Delta x\left(i=0,1, \ldots, N_{1}\right)$ and $y_{j}=y_{L}+j \Delta y\left(j=0,1, \ldots, N_{2}\right)$, respectively. For simplicity, we denote $U_{i, j}^{n}=U\left(x_{i}, y_{j}, t_{n}\right)$ and

$$
\begin{aligned}
& \delta_{x}^{2} U_{i, j}^{n}=\frac{U_{i+1, j}^{n}-2 U_{i, j}^{n}+U_{i-1, j}^{n}}{\Delta x^{2}}, \quad \delta_{y}^{2} U_{i, j}^{n}=\frac{U_{i, j+1}^{n}-2 U_{i, j}^{n}+U_{i, j-1}^{n}}{\Delta y^{2}}, \\
& \delta_{x} U_{i+1 / 2, j}^{n}=\frac{U_{i+1, j}^{n}-U_{i, j}^{n}}{\Delta x}, \quad \delta_{y} U_{i, j+1 / 2}^{n}=\frac{U_{i, j+1}^{n}-U_{i, j}^{n}}{\Delta y} .
\end{aligned}
$$

In all the schemes throughout this paper, we use the second-order time discretization developed in [44] (see also the related work in [45]), which is based on Lubich's fractional linear multi-step methods [47]. We first review the second-order fractional linear multistep methods developed in [44] for the time discretization of (1). To illustrate the idea of this discretization, we consider the following fractional ordinary differential equation (FODE)

$$
{ }_{C} D_{0, t}^{\beta} y(t)=\mu y(t)+g(t), \quad y(0)=y_{0}, \quad 0<\beta<1 .
$$

Suppose that $y(t)$ is suitably smooth. Two second-order methods for (4) are given by [44]

$$
\begin{aligned}
\frac{1}{\tau^{\beta}} \sum_{k=0}^{n} \omega_{n-k}\left(y\left(t_{k}\right)-y_{0}\right)= & \mu \sum_{k=0}^{n} \theta_{n-k}^{(q)} y\left(t_{k}\right)+\mu B_{n}^{(q)} y_{0}+\mu C_{n}^{(q)}\left(y\left(t_{1}\right)-y_{0}\right) \\
& +\frac{1}{\tau^{\beta}} \sum_{k=0}^{n} \omega_{n-k}\left[D_{0, t}^{-\beta} g(t)\right]_{t=t_{k}}+O\left(\tau^{2}\right),
\end{aligned}
$$

where $q=1,2$, and $B_{n}^{(q)}$ and $C_{n}^{(q)}$ are defined by

$$
\begin{aligned}
B_{n}^{(q)} & =\frac{1}{\Gamma(1+\beta)} \sum_{k=0}^{n} \omega_{n-k} k^{\beta}-\sum_{k=0}^{n} \theta_{k}^{(q)}, \\
C_{n}^{(q)} & =\frac{\Gamma(2)}{\Gamma(2+\beta)} \sum_{k=0}^{n} \omega_{n-k} k^{1+\beta}-\sum_{k=1}^{n} \theta_{n-k}^{(q)} k,
\end{aligned}
$$

with $\omega_{k}$ and $\theta_{k}^{(q)}(q=1,2)$ given as follows

$$
\begin{aligned}
& \omega_{k}=(-1)^{k}\left(\begin{array}{l}
\beta \\
k
\end{array}\right)=\frac{\Gamma(k-\beta)}{\Gamma(-\beta) \Gamma(k+1)}, \\
& \theta_{k}^{(1)}=2^{-\beta}(-1)^{k} \omega_{k}, k \geq 0 ; \quad \theta_{0}^{(2)}=1-\frac{\beta}{2}, \theta_{1}^{(2)}=\frac{\beta}{2}, \theta_{k}^{(2)}=0, k>1 .
\end{aligned}
$$

For simplicity, we introduce the following notations

$$
\begin{aligned}
& D^{(n)} U=\frac{1}{\tau^{\beta}} \sum_{k=0}^{n} \omega_{k}\left(U^{n-k}-U^{0}\right)=\frac{1}{\tau^{\beta}}\left[\sum_{k=0}^{n} \omega_{k} U^{n-k}-b_{n} U^{0}\right], \\
& L_{q}^{(n)} U=\sum_{k=0}^{n} \theta_{k}^{(q)} U^{n-k}, \quad q=1,2,
\end{aligned}
$$


where $\omega_{k}$ and $\theta_{k}^{(q)}(q=1,2)$ are defined by (8) and (9), respectively, and $b_{n}$ is given by

$$
b_{n}=\sum_{k=0}^{n} \omega_{k}=\frac{\Gamma(n+1-\beta)}{\Gamma(1-\beta) \Gamma(n+1)} . \quad n \geq 0,
$$

From (5), the semi-discretization (time discretization) for (1) reads

$$
D^{(n)} U=\mu L_{q}^{(n)} \Delta U+\mu B_{n}^{(q)} \Delta U^{0}+\mu C_{n}^{(q)} \Delta\left(U^{1}-U^{0}\right)+\frac{1}{\tau^{\beta}} F^{n}+O\left(\tau^{2}\right),
$$

where $D^{(n)}$ and $L_{q}^{(n)}$ are defined by $(10),(11)$, respectively, $B_{n}^{(q)}$ and $C_{n}^{(q)}$ are defined by (6) and (7), respectively, and $F^{n}$ is given by

$$
F^{n}=\sum_{k=0}^{n} \omega_{n-k}\left[D_{0, t}^{-\beta} f(x, y, t)\right]_{t=t_{k}},
$$

in which $\omega_{k}$ is defined by (8).

\subsection{Derivations of ADI finite difference methods}

Based on the time discretization (13), we are ready to develop the two ADI FDMs for (1). Adding the perturbation term $\left(\theta_{0}^{(q)}\right)^{2} \mu^{2} \tau^{\beta} \partial_{x}^{2} \partial_{y}^{2}\left(U^{n}-U^{n-1}\right)=O\left(\tau^{1+\beta}\right)$ for $n>1$ to both sides of Eq. (13) yields

$$
\begin{aligned}
& D^{(n)} U+\left(\theta_{0}^{(q)}\right)^{2} \mu^{2} \tau^{\beta} \partial_{x}^{2} \partial_{y}^{2}\left(U^{n}-U^{n-1}\right) \\
= & \mu L_{q}^{(n)} \Delta U+\mu B_{n}^{(q)} \Delta U^{0}+\mu C_{n}^{(q)} \Delta\left(U^{1}-U^{0}\right)+\frac{1}{\tau^{\beta}} F^{n}+O\left(\tau^{1+\beta}\right),
\end{aligned}
$$

where $D^{(n)}, L_{q}^{(n)}, B_{n}^{(q)}, C_{n}^{(q)}$, and $F^{n}$ are defined by (10), (11), (6), (7), and (14), respectively. If $n=1$ in (13), then we can add $\mu^{2} \tau^{\beta}\left(\theta_{0}^{(q)}+C_{1}^{(q)}\right)^{2} \partial_{x}^{2} \partial_{y}^{2}\left(U^{1}-U^{0}\right)=O\left(\tau^{1+\beta}\right)$ to both sides of (13) to obtain

$$
\begin{aligned}
& D^{(n)} U+\mu^{2} \tau^{\beta}\left(2^{-\beta}+C_{1}^{(q)}\right)^{2} \partial_{x}^{2} \partial_{y}^{2}\left(U^{1}-U^{0}\right) \\
= & \mu L_{q}^{(n)} \Delta U+\mu B_{n}^{(q)} \Delta U^{0}+\mu C_{n}^{(q)} \Delta\left(U^{1}-U^{0}\right)+\frac{1}{\tau^{\beta}} F^{1}+O\left(\tau^{1+\beta}\right) .
\end{aligned}
$$

From (15)-(16), we obtain two schemes for (1) which can be readily written as ADI FDMs:

- ADI FDM $(q)$ : Find $u_{i, j}^{n}\left(0<i<N_{1}, 0<j<N_{2}\right)$ for $n=1,2, \ldots, n_{T}$, such that

$$
\begin{aligned}
& D^{(n)} u_{i, j}+\mu^{2} \tau^{\beta}\left(\theta_{0}^{(q)}+\delta_{n, 1} C_{1}^{(q)}\right)^{2} \delta_{x}^{2} \delta_{y}^{2}\left(u_{i, j}^{n}-u_{i, j}^{n-1}\right) \\
= & \mu L_{q}^{(n)}\left(\delta_{x}^{2}+\delta_{y}^{2}\right) u_{i, j}+\mu B_{n}^{(q)}\left(\delta_{x}^{2}+\delta_{y}^{2}\right) u_{i, j}^{0}+\mu C_{n}^{(q)}\left(\delta_{x}^{2}+\delta_{y}^{2}\right)\left(u_{i, j}^{1}-u_{i, j}^{0}\right)+\frac{1}{\tau^{\beta}} F_{i, j}^{n},
\end{aligned}
$$

where $D^{(n)}, L_{q}^{(n)}, B_{n}^{(q)}, C_{n}^{(q)}$, and $F^{n}$ are defined by (10), (11), (6), (7), and (14), respectively, $\delta_{1,1}=1$ and $\delta_{n, 1}=0$ for $n \neq 1, \theta_{0}^{(1)}=2^{-\beta}$, and $\theta_{0}^{(2)}=1-\beta / 2$. The initial and boundary conditions are given by

$$
\begin{aligned}
& u_{i, j}^{0}=\phi_{0}\left(x_{i}, y_{j}\right), \quad 0 \leq i \leq N_{1}, 0 \leq j \leq N_{2}, \\
& u_{0, j}^{k}=u_{N_{1}, j}^{k}=u_{i, 0}^{k}=u_{i, N_{2}}^{k}=0, \quad 0 \leq i \leq N_{1}, 1 \leq j \leq N_{2}-1,1 \leq k \leq n_{T} .
\end{aligned}
$$


For $n>1$, as in [3], the difference scheme (17) can be written as

$$
\left(1+\mu_{1} \delta_{x}^{2}\right)\left(1+\mu_{2} \delta_{y}^{2}\right) u_{i, j}^{n}=(R H S)_{i, j}^{n}
$$

where

$$
\begin{aligned}
(R H S)_{i, j}^{n}= & \sum_{k=1}^{n}\left[-\omega_{k} u_{i, j}^{n-k}+\mu \tau^{\beta} \theta_{k}^{(q)}\left(\delta_{x}^{2}+\delta_{y}^{2}\right) u_{i, j}^{n-k}\right]+\mu \tau^{\beta} B_{n}^{(q)}\left(\delta_{x}^{2}+\delta_{y}^{2}\right) u_{i, j}^{0} \\
& +b_{n} u_{i, j}^{0}+\mu \tau^{\beta} C_{n}^{(q)}\left(\delta_{x}^{2}+\delta_{y}^{2}\right)\left(u_{i, j}^{1}-u_{i, j}^{0}\right)+F_{i, j}^{n}+\left(\theta_{0}^{(q)} \mu \tau^{\beta}\right)^{2} \delta_{x}^{2} \delta_{y}^{2} u_{i, j}^{n-1} .
\end{aligned}
$$

Eq. (19) can be solved by the following two steps [3]

1) For each $j\left(1 \leq j \leq N_{2}-1\right)$, solve $\left(1+\mu_{1} \delta_{x}^{2}\right) u_{i, j}^{*}=(R H S)_{i, j}^{n}, \quad 1 \leq i \leq N_{1}-1$;

2) For each $i\left(1 \leq i \leq N_{1}-1\right)$, solve $\left(1+\mu_{2} \delta_{y}^{2}\right) u_{i, j}^{n}=u_{i, j}^{*}, \quad 1 \leq j \leq N_{2}-1$;

here the boundary conditions of the first equation of the above equation are given by $u_{0, j}^{*}=$ $\left(1+\mu_{2} \delta_{y}^{2}\right) u_{0, j}^{n}, u_{N_{1}, j}^{*}=\left(1+\mu_{2} \delta_{y}^{2}\right) u_{N_{1}, j}^{n}$.

In our computation and theoretical analysis, we use (19), the matrix representation of which is given by

$$
\left(E_{N_{1}-1}+\mu_{1} S_{N_{1}-1}\right) \underline{\mathbf{u}}^{n}\left(E_{N_{2}-1}+\mu_{2} S_{N_{2}-1}\right)=\underline{\mathbf{b}}^{n}, \quad n>1,
$$

where $E_{N}$ is an $N \times N$ identity matrix, $\mu_{1}=\frac{\theta_{0}^{(q)} \mu \tau^{\beta}}{\Delta x^{2}}, \mu_{2}=\frac{\theta_{0}^{(q)} \mu \tau^{\beta}}{\Delta y^{2}},\left(\underline{\mathbf{u}}^{n}\right)_{i-1, j-1}=u_{i, j}, i=$ $1,2, \ldots, N_{1}-1, j=1,2, \ldots, N_{2}-1$, and the tridiagonal matrix $S_{N} \in \mathbb{R}^{N \times N}$ is defined by

$$
S_{N}=\left(\begin{array}{cccccc}
2 & -1 & 0 & \cdots & 0 & 0 \\
-1 & 2 & -1 & \cdots & 0 & 0 \\
0 & -1 & 2 & \cdots & 0 & 0 \\
\vdots & \vdots & \vdots & \ddots & \vdots & \vdots \\
0 & 0 & 0 & \cdots & 2 & -1 \\
0 & 0 & 0 & \cdots & -1 & 2
\end{array}\right)_{N \times N}
$$

and the right-hand-side matrix $\underline{\mathbf{b}}^{n} \in \mathbb{R}^{\left(N_{1}-1\right) \times\left(N_{2}-1\right)}$ in (20) is given by

$$
\left(\underline{\mathbf{b}}^{n}\right)_{i-1, j-1}=(R H S)_{i, j}^{n}, \quad i=1,2, \ldots, N_{1}-1, j=1,2, \ldots, N_{2}-1 \text {. }
$$

Hence, the matrix equation (20) can be solved with two steps in the ADI method: 1) solve $\left(E_{N_{1}-1}+\mu_{1} S_{N_{1}-1}\right) \mathbf{u}^{*}=\underline{\mathbf{b}}^{n}$ to get $\mathbf{u}^{*}$ with $O\left(N_{1} N_{2}\right)$ operations; 2$)$ then solve $\left(E_{N_{2}-1}+\right.$ $\left.\mu_{2} S_{N_{2}-1}\right) \mathbf{u}^{* *}=\left(\mathbf{u}^{*}\right)^{T}$ to obtain $\underline{\mathbf{u}}^{n}=\left(\mathbf{u}^{* *}\right)^{T}$ with $O\left(N_{1} N_{2}\right)$ operations. Hence, the computational complexity of ADI method (17) in physical space is $O\left(N_{1} N_{2}\right)$. 


\subsection{Stability and convergence}

Next, we study the stability and convergence of the ADI schemes (17). Define the discrete inner product $(\cdot, \cdot)$ and norm $\|\cdot\|$ as

$$
(\mathbf{u}, \mathbf{v})=\Delta x \Delta y \sum_{i=0}^{N_{1}-1} \sum_{j=0}^{N_{2}-1} u_{i, j} v_{i, j}, \quad\|\mathbf{u}\|=\sqrt{(\mathbf{u}, \mathbf{u})}
$$

where $\mathbf{u}, \mathbf{v} \in \mathbb{R}^{\left(N_{1}+1\right) \times\left(N_{2}+1\right)}$ with $(\mathbf{u})_{i, j}=u_{i, j},(\mathbf{v})_{i, j}=v_{i, j}\left(0 \leq i \leq N_{1}, 0 \leq j \leq N_{2}\right)$. For $\mathbf{u}, \mathbf{v} \in \mathbb{R}^{\left(N_{1}+1\right) \times\left(N_{2}+1\right)}$ with $u_{i, 0}=u_{i, N_{2}}=u_{0, j}=u_{N_{1}, j}=0$ and $v_{i, 0}=v_{i, N_{2}}=v_{0, j}=v_{N_{1}, j}=0$, we can readily derive

$$
\left(\delta_{x}^{2} \mathbf{u}, \mathbf{v}\right)=-\left(\delta_{x} \mathbf{u}, \delta_{x} \mathbf{v}\right), \quad\left(\delta_{y}^{2} \mathbf{u}, \mathbf{v}\right)=-\left(\delta_{y} \mathbf{u}, \delta_{y} \mathbf{v}\right),
$$

where we define that $\left(\delta_{x}^{2} \mathbf{u}\right)_{0, j}=\left(\delta_{x}^{2} \mathbf{u}\right)_{N_{1}, j}=0$ for $0 \leq j \leq N_{2},\left(\delta_{x}^{2} \mathbf{u}\right)_{i, j}=\delta_{x}^{2} u_{i, j}^{n}(0<i<$ $\left.N_{1}, 0<j<N_{2}\right),\left(\delta_{x} \mathbf{u}\right)_{N_{1}, j}=0$ for $0 \leq j \leq N_{2},\left(\delta_{x} \mathbf{u}\right)_{i, j}=\delta_{x} u_{i+1 / 2, j}^{n}\left(0 \leq i<N_{1}, 0 \leq j \leq N_{2}\right)$; $\delta_{y}^{2} \mathbf{u}$ and $\delta_{y} \mathbf{u}$ are defined similarly.

For convenience, we define the norms $\left|\|\cdot \mid\| \|_{q}(q=1,2)\right.$ as

$$
\|\mid \mathbf{u}\|_{q}=\left(\|\mathbf{u}\|^{2}+\mu \tau^{\beta} \theta_{0}^{(q)}\left(\left\|\delta_{x} \mathbf{u}\right\|^{2}+\left\|\delta_{y} \mathbf{u}\right\|^{2}\right)\right)^{1 / 2} .
$$

Next, we present stability and convergence for ADI FDM $(q)$.

Theorem 2.1. Suppose that $u_{i, j}^{k}\left(i=0,1,2, \ldots, N_{1}, j=0,1, \ldots, N_{2}\right)$ for $k=1,2, \ldots, n_{T}$ is the solution of (17). Then, there exists a positive constant $C$ independent of $n, \Delta x, \Delta y, \tau$, such that

$$
\tau \sum_{k=1}^{n}\left\|\left|\mathbf{u}^{k} \|\right|_{q}^{2} \leq C\left(\left\|\mid \mathbf{u}^{0}\right\|\left\|_{l}^{2}+\tau^{1+\beta}\right\| \delta_{x} \delta_{y} \mathbf{u}^{0}\left\|^{2}+\max _{0 \leq t \leq t_{n}}\right\| \mathbf{f}(t) \|^{2}\right), \quad q=1 \text { or } 2,\right.
$$

where $\left(\mathbf{u}^{k}\right)_{i, j}=u_{i, j}^{n}$ and $(\mathbf{f}(t))_{i, j}=f\left(x_{i}, y_{j}, t\right)$.

Theorem 2.2. Suppose that $U$ and $u_{i, j}^{n}\left(0 \leq i \leq N_{1}, 0 \leq j \leq N_{2}, 1 \leq n \leq n_{T}\right)$ are the solutions to (1) and (17), respectively. If $U \in C^{2}\left(0, T ; C^{4}(\Omega)\right), f \in C(0, T ; C(\Omega))$ and $\phi_{0} \in C(\bar{\Omega})$, then there exists a positive constant $C$ independent of $n, \Delta x, \Delta y$ and $\tau$, such that

$$
\sqrt{\tau \sum_{k=1}^{n}\left\|\mathbf{u}^{k}-\mathbf{U}\left(t_{k}\right)\right\|^{2}} \leq C\left(\tau^{1+\beta}+\Delta x^{2}+\Delta y^{2}\right)
$$

where $\left(\mathbf{U}\left(t_{k}\right)\right)_{i, j}=U\left(x_{i}, y_{j}, t_{k}\right)$.

The perturbation terms in the ADI methods, see e.g. $\left(\theta_{0}^{(q)}\right)^{2} \mu^{2} \tau^{\beta} \partial_{x}^{2} \partial_{y}^{2}\left(U^{n}-U^{n-1}\right)$ in (15), may lead to unsatisfactory accuracy, especially when $\beta$ is small and/or the analytical solution $U(x, y, t)$ is steep in space (see numerical results in Tables 3 and 4 in Section 5). In the following sections, we mainly focus on the non-ADI FDMs with fast solvers such that the computational cost in space is $O\left(N^{2} \log (N)\right)$ for two-dimensional subdiffusion while the high-order accuracy is maintained. The ADI method (17) was introduced in [30] without a detailed theoretical analysis. Here we present the stability and convergence analysis to address the difference between non-ADI difference schemes. 


\section{Fast difference schemes based on fast Possion solver}

In this section, we first present two fully discrete non-ADI finite difference schemes for (1). Then, we provide a fast Poisson solver to solve the linear system derived from the two difference schemes. Afterwards, we prove the stability and convergence of the two schemes. Lastly, the two compact difference schemes with a fast solver are constructed.

\subsection{Central difference in space}

In the semi-discrete approximation (13) of (1), we can apply the central difference in space or drop the perturbation term $\mu^{2} \tau^{\beta}\left(\theta_{0}^{(q)}+\delta_{n, 1} C_{1}^{(q)}\right)^{2} \delta_{x}^{2} \delta_{y}^{2}\left(u_{i, j}^{n}-u_{i, j}^{n-1}\right)$ in (17) to obtain the non-ADI FDMs for (1) as follows:

- $\operatorname{FDM~I~}(q)$ : Find $u_{i, j}^{n}\left(0<i<N_{1}, 0<j<N_{2}\right)$ for $n=1,2, \ldots, n_{T}$, such that

$$
D^{(n)} u_{i, j}=\mu L_{q}^{(n)}\left(\delta_{x}^{2}+\delta_{y}^{2}\right) u_{i, j}+\mu B_{n}^{(q)}\left(\delta_{x}^{2}+\delta_{y}^{2}\right) u_{i, j}^{0}+\mu C_{n}^{(q)}\left(\delta_{x}^{2}+\delta_{y}^{2}\right)\left(u_{i, j}^{1}-u_{i, j}^{0}\right)+\frac{1}{\tau^{\beta}} F_{i, j}^{n},
$$

where $D^{(n)}, L_{q}^{(n)}, B_{n}^{(q)}, C_{n}^{(q)}$, and $F^{n}$ are defined by (10), (11), (6), (7), and (14), respectively. The initial and boundary conditions are given by (18).

We extend the fast Poisson solver [48] to solve the scheme (24) efficiently. We first write the matrix representation of (24) as

$$
\underline{\mathbf{u}}^{n}+\mu_{1} S_{N_{1}-1} \underline{\mathbf{u}}^{n}+\mu_{2} \underline{\mathbf{u}}^{n} S_{N_{2}-1}=\underline{\mathbf{b}}^{n},
$$

where $\mu_{1}=\frac{\theta_{0}^{(q)} \mu \tau^{\beta}}{\Delta x^{2}}, \mu_{2}=\frac{\theta_{0}^{(q)} \mu \tau^{\beta}}{\Delta y^{2}},\left(\underline{\mathbf{u}}^{n}\right)=u_{i, j}, i=1,2, \ldots, N_{1}-1, j=1,2, \ldots, N_{2}-1$, the matrix $S_{N} \in \mathbb{R}^{N \times N}$ is from (21), and the matrix $\underline{\mathbf{b}}^{n} \in \mathbb{R}^{\left(N_{1}-1\right) \times\left(N_{2}-1\right)}$ in (25) is given by

$$
\begin{aligned}
\left(\underline{\mathbf{b}}^{n}\right)_{i-1, j-1}= & \sum_{k=1}^{n}\left[-\omega_{k} u_{i, j}^{n-k}+\mu \tau^{\beta} \theta_{k}^{(q)}\left(\delta_{x}^{2}+\delta_{y}^{2}\right) u_{i, j}^{n-k}\right]+b_{n} u_{i, j}^{0}+\mu \tau^{\beta} B_{n}^{(q)}\left(\delta_{x}^{2}+\delta_{y}^{2}\right) u_{i, j}^{0} \\
& +\mu \tau^{\beta} C_{n}^{(q)}\left(\delta_{x}^{2}+\delta_{y}^{2}\right)\left(u_{i, j}^{1}-u_{i, j}^{0}\right)+F_{i, j}^{n}, \quad i=1,2, \ldots, N_{1}-1, j=1,2, \ldots, N_{2}-1 .
\end{aligned}
$$

Remark 3.1. We assume that FDM I (q) satisfies homogenous boundary conditions, which leads to (25). If we impose nonhomogenous boundary conditions to (1), then FDM I (q) still holds. In such a case, (25) becomes $\underline{\mathbf{u}}^{n}+\mu_{1} S_{N_{1}-1} \underline{\mathbf{u}}^{n}+\mu_{2} \underline{\mathbf{u}}^{n} S_{N_{2}-1}=\underline{\mathbf{B}}^{n}$, where $\underline{\mathbf{B}}^{n}$ satisfies

$$
\underline{\mathbf{B}}^{n}=\underline{\mathbf{b}}^{n}+\mu_{1}\left(\begin{array}{cccc}
u_{0,1}^{n} & u_{0,2}^{n} & \cdots & u_{0, N_{2}-1}^{n} \\
0 & 0 & \cdots & 0 \\
\vdots & \vdots & \ddots & \vdots \\
0 & 0 & \cdots & 0 \\
u_{N_{1}, 1}^{n} & u_{N_{1}, 2}^{n} & \cdots & u_{N_{1}, N_{2}-1}^{n}
\end{array}\right)+\mu_{2}\left(\begin{array}{ccccc}
u_{1,0}^{n} & 0 & \cdots & 0 & u_{1, N_{2}}^{n} \\
u_{2,0}^{n} & 0 & \cdots & 0 & u_{2, N_{2}}^{n} \\
\vdots & \vdots & \ddots & \vdots & \vdots \\
u_{N_{1}-1,0}^{n} & 0 & \cdots & 0 & u_{N_{1}-1, N_{2}}^{n}
\end{array}\right) \text {. }
$$


Now we can employ a fast solver technique from [48] to effectively solve the matrix equation (25). Let $\lambda_{j}^{(k)}$ and $\mathbf{q}_{j}^{(k)}$ be the $j$-th eigenvalue and eigenvector of $S_{N_{k}-1}, N_{k}(k=$ $1,2, \ldots)$ is a positive integer. Then we have

$$
S_{N_{k}-1} Q^{(k)}=Q^{(k)} \Lambda^{(k)}, \quad\left(Q^{(k)}\right)^{T} Q^{(k)}=\left(Q^{(k)}\right)^{2}=\frac{1}{2 h_{k}} E_{N_{k}-1},
$$

where $Q^{(k)}=\left[\mathbf{q}_{1}^{(k)}, \mathbf{q}_{2}^{(k)}, \ldots, \mathbf{q}_{N_{k}-1}^{(k)}\right], \Lambda^{(k)}=\operatorname{diag}\left(\lambda_{1}^{(k)}, \lambda_{2}^{(k)}, \ldots, \lambda_{N_{k}-1}^{(k)}\right)$, and $E_{N_{k}}$ is an $N_{k} \times N_{k}$ identity matrix. Also, we have explicit representation of $\lambda_{j}^{(k)}$ and $\mathbf{q}_{j}^{(k)}$ (see e.g. [49]):

$$
\begin{aligned}
& \lambda_{j}^{(k)}=4 \sin ^{2}\left(\frac{j \pi h_{k}}{2}\right), \quad h_{k}=1 / N_{k}, \quad j=1,2, \ldots, N_{k}-1, \\
& \mathbf{q}_{j}^{(k)}=\left(\sin \left(j \pi h_{k}\right), \sin \left(2 j \pi h_{k}\right), \cdots, \sin \left(\left(N_{k}-1\right) j \pi h_{k}\right)\right)^{T} .
\end{aligned}
$$

Define $V \in \mathbb{R}^{\left(N_{1}-1\right) \times\left(N_{2}-1\right)}$ such that

$$
\underline{\mathbf{u}}^{n}=Q^{(1)} V Q^{(2)} \text {. }
$$

By (26) and (27), (25) is equivalent to

$$
V+\mu_{1} \Lambda^{(1)} V+\mu_{2} V \Lambda^{(2)}=4 h_{1} h_{2} Q^{(1)} \underline{\mathbf{b}}^{n} Q^{(2)}=R .
$$

The linear system (29) can be solved simply by

$$
V_{i, j}=\frac{R_{i, j}}{1+\mu_{1} \lambda_{i}^{(1)}+\mu_{2} \lambda_{j}^{(2)}}, \quad i=1,2, \ldots, N_{1}-1, j=1,2, \ldots, N_{2}-1 .
$$

Once $V$ is obtained, we can obtain the solution $\underline{\mathbf{u}}^{n}$ from (28).

Note that $R$ in the right-hand side of (29) can be computed with $O\left(N_{1} N_{2} \log \left(N_{1}\right)\right)+$ $O\left(N_{1} N_{2} \log \left(N_{2}\right)\right)$ operations using the fast Fourier transform. Also, $\underline{\mathbf{u}}^{n}=Q^{(1)} V Q^{(2)}$ can be computed similarly. In conclusion, we can obtain the solution to (25) from (28)-(30) with $O\left(N_{1} N_{2} \log \left(N_{1}\right)\right)+O\left(N_{1} N_{2} \log \left(N_{2}\right)\right)$ operations.

We now present the stability and convergence for the schemes (24), the proof of which is left in Appendix.

Theorem 3.1 (Stability). Suppose that $u_{i, j}^{k}\left(i=1,2, \ldots, N_{1}-1, j=1,2, \ldots, N_{2}-1\right)$ for $k=1,2, \ldots, n_{T}$ is the solution of $(24), u_{i, 0}^{k}=u_{i, N_{2}}^{k}=u_{0, j}^{k}=u_{N_{1}, j}^{k}=0$. Then, there exist positive constants $C_{1}$ independent of $n, \Delta x, \Delta y, \tau$ and $T$, and $C_{2}$ independent of $n, \Delta x, \Delta y$, and $\tau$ such that

$$
\left\|\left.\left|\mathbf{u}^{n}\right|\right|_{q} ^{2} \leq C_{1}\left|\left\|\mathbf{u}^{0} \mid\right\|_{q}^{2}+C_{2} \max _{0 \leq t \leq T}\|\mathbf{f}(t)\|^{2}, \quad q=1,2,\right.\right.
$$

where $\mathbf{u}^{k}, \mathbf{f}(t) \in \mathbb{R}^{\left(N_{1}+1\right) \times\left(N_{2}+1\right)}$ with $\left(\mathbf{u}^{k}\right)=u_{i, j}^{k}$ and $(\mathbf{f}(t))=f\left(x_{i}, y_{j}, t\right)$.

By Theorem 3.1, we can readily obtain the following convergence theorem.

Theorem 3.2 (Convergence). Suppose that $U$ and $u_{i, j}^{n}\left(0 \leq i \leq N_{1}, 0 \leq j \leq N_{2}, 1 \leq n \leq n_{T}\right)$ are the solutions to (1) and (24), respectively. If $U \in C^{2}\left(0, T ; C^{4}(\Omega)\right), f \in C(0, T ; C(\Omega))$ and $\phi_{0} \in C(\bar{\Omega})$, then there exists a positive constant $C$ independent of $n, \Delta x, \Delta y$ and $\tau$, such that

$$
\left\|\mathbf{u}^{n}-\mathbf{U}\left(t_{n}\right)\right\| \leq C\left(\tau^{2}+\Delta x^{2}+\Delta y^{2}\right) .
$$




\subsection{Compact difference in space}

In this subsection, we apply higher-order compact finite difference schemes in physical space for (1). The fourth-order compact finite difference method for the model problem $\left(\partial_{x}^{2}+\partial_{y}^{2}\right) U=f(x, y)$ with homogeneous boundary conditions is given by: Find $u_{i, j}$ such that

$$
\mathcal{H} u_{i, j}=\mathcal{A} f_{i, j}, \quad 0<i<N_{1}, 0<j<N_{2},
$$

where $\mathcal{A}$ and $\mathcal{H}$ are defined by

$$
\begin{aligned}
& \mathcal{A} u_{i, j}=u_{i, j}+\frac{1}{12}\left(\Delta x^{2} \delta_{x}^{2}+\Delta y^{2} \delta_{y}^{2}\right) u_{i, j}, \quad 0<i<N_{1}, 0<j<N_{2}, \\
& \mathcal{H} u_{i, j}=\left(\delta_{x}^{2}+\delta_{y}^{2}\right) u_{i, j}+\frac{1}{12}\left(\Delta x^{2}+\Delta y^{2}\right) \delta_{x}^{2} \delta_{y}^{2} u_{i, j}, \quad 0<i<N_{1}, 0<j<N_{2} .
\end{aligned}
$$

The fourth-order truncation error $O\left(\Delta x^{4}+\Delta y^{4}+\Delta x^{2} \Delta y^{2}\right)$ can be verified by the Taylor's expansion. for $(1)$ :

By (13) and (33), we obtain the following compact finite difference methods (CFDMs)

- $\operatorname{CFDM}$ I $(q)$ : Find $u_{i, j}^{n}\left(0<i<N_{1}, 0<j<N_{2}\right)$ for $n=1,2, \ldots, n_{T}$, such that

$$
D^{(n)} \mathcal{A} u_{i, j}=\mu L_{q}^{(n)} \mathcal{H} u_{i, j}+\mu B_{n}^{(q)} \mathcal{H} u_{i, j}^{0}+\mu C_{n}^{(q)}\left(\mathcal{H} u_{i, j}^{1}-\mathcal{H} u_{i, j}^{0}\right)+\frac{1}{\tau^{\beta}} \mathcal{A} F_{i, j}^{n},
$$

where $D^{(n)}, L_{q}^{(n)}, B_{n}^{(q)}, C_{n}^{(q)}$, and $F^{n}$ are defined by (10), (11), (6), (7), and (14), respectively. The initial and boundary conditions for (34) are taken as in (18).

We rewrite the matrix representation of (34) as

$$
\underline{\mathbf{u}}^{n}-\frac{1}{12}\left(S_{N_{1}-1} \underline{\mathbf{u}}^{n}+\underline{\mathbf{u}}^{n} S_{N_{2}-1}\right)+\mu_{1} S_{N_{1}-1} \underline{\mathbf{u}}^{n}+\mu_{2} \underline{\mathbf{u}}^{n} S_{N_{2}-1}-\mu_{3} S_{N_{1}-1} \underline{\mathbf{u}}^{n} S_{N_{2}-1}=\underline{\mathbf{b}}^{n}
$$

where $\mu_{1}=\frac{\theta_{0}^{(q)} \mu \tau^{\beta}}{\Delta x^{2}}, \mu_{2}=\frac{\theta_{0}^{(q)} \mu \tau^{\beta}}{\Delta y^{2}}, \mu_{3}=\frac{\theta_{0}^{(q)} \mu \tau^{\beta}\left(\Delta x^{2}+\Delta y^{2}\right)}{12 \Delta x^{2} \Delta y^{2}}, S_{N}$ is defined by (21), and $\underline{\mathbf{b}}^{n} \in$ $\mathbb{R}^{\left(N_{1}-1\right) \times\left(N_{2}-1\right)}$ is defined by

$$
\begin{aligned}
\left(\underline{\mathbf{b}}^{n}\right)_{i-1, j-1}= & \sum_{k=0}^{n}\left[\omega_{k} \mathcal{A}\left(u_{i, j}^{n-k}-u_{i, j}^{0}\right)+\mu \tau^{\beta} \theta_{k}^{(q)} \mathcal{H} u_{i, j}^{n-k}\right]+\mu \tau^{\beta} B_{n}^{(q)} \mathcal{H} u_{i, j}^{0} \\
& +\mu \tau^{\beta} C_{n}^{(q)} \mathcal{H}\left(u_{i, j}^{1}-u_{i, j}^{0}\right)+\mathcal{A} F_{i, j}^{n}, \quad i=1,2, \ldots, N_{1}-1, j=1,2, \ldots, N_{2}-1 .
\end{aligned}
$$

Let $\underline{\mathbf{u}}^{n}=Q^{(1)} V Q^{(2)}$. Then similar to (29), we can obtain

$$
V+\left(\mu_{1}-1 / 12\right) \Lambda^{(1)} V+\left(\mu_{2}-1 / 12\right) V \Lambda^{(2)}-\mu_{3} \Lambda^{(1)} V \Lambda^{(2)}=4 h_{1} h_{2} Q^{(1)} \underline{\mathbf{b}}^{n} Q^{(2)}=R .
$$

or equivalently, for $i=1,2, \ldots, N_{1}-1, j=1,2, \ldots, N_{2}-1$

$$
V_{i, j}=\frac{R_{i, j}}{1+\left(\mu_{1}-1 / 12\right) \lambda_{i}^{(1)}+\left(\mu_{2}-1 / 12\right) \lambda_{j}^{(2)}-\mu_{3} \lambda_{i}^{(1)} \lambda_{j}^{(2)}} .
$$


Therefore, the matrix equation (35) can be solved with complexity of $O\left(N_{1} N_{2} \log \left(N_{1}\right)\right)+$ $O\left(N_{1} N_{2} \log \left(N_{2}\right)\right)$ operations as that of $(25)$.

Define the norms $\||\cdot|\|_{3}$ and $\||\cdot|\|_{4}$ as

$$
\|\mathbf{u}\|\left\|_{3}=\left(\|\mathbf{u}\|_{\mathcal{A}}^{2}+\mu \theta_{0}^{(1)} \tau^{\beta}\|\mathbf{u}\|_{\mathcal{H}}^{2}\right)^{1 / 2}, \quad\right\|\|\mathbf{u}\|_{4}=\left(\|\mathbf{u}\|_{\mathcal{A}}^{2}+\mu \theta_{0}^{(2)} \tau^{\beta}\|\mathbf{u}\|_{\mathcal{H}}^{2}\right)^{1 / 2}
$$

where

$$
\begin{aligned}
& \|\mathbf{u}\|_{\mathcal{A}}=\sqrt{(\mathbf{u}, \mathbf{u})_{\mathcal{A}}}, \quad(\mathbf{u}, \mathbf{v})_{\mathcal{A}}=\Delta x \Delta y \sum_{i=0}^{N_{1}-1} \sum_{j=0}^{N_{2}-1}\left(\mathcal{A} u_{i, j}\right) v_{i, j}, \\
& \|\mathbf{u}\|_{\mathcal{H}}=\sqrt{(\mathbf{u}, \mathbf{u})_{\mathcal{H}}}, \quad(\mathbf{u}, \mathbf{v})_{\mathcal{H}}=-\Delta x \Delta y \sum_{i=0}^{N_{1}-1} \sum_{j=0}^{N_{2}-1}\left(\mathcal{H} u_{i, j}\right) v_{i, j},
\end{aligned}
$$

in which $\mathbf{u}, \mathbf{v} \in \mathcal{V}_{0}=\left\{\mathbf{u}: \mathbf{u} \in \mathbb{R}^{\left(N_{1}+1\right) \times\left(N_{2}+1\right)},(\mathbf{u})_{i, j}=u_{i, j}\right.$ with $u_{i, j}=0$ for $i=0$ or $i=$ $N_{1}$ or $j=0$, or $j=N_{2}$. We also let $\mathcal{A} u_{i, j}=\mathcal{H} u_{i, j}=0$ for $i=0, N_{1}$ or $j=0, N_{2}$. We will illustrate that $(\cdot, \cdot)_{\mathcal{A}}$ and $(\cdot, \cdot)_{\mathcal{H}}$ are two kinds of inner products in Appendix.

Next, we present the following stability and convergence results, the proofs of which are given in Appendix.

Theorem 3.3 (Stability). Suppose that $u_{i, j}^{k}\left(i=1,2, \ldots, N_{1}-1, j=1,2, \ldots, N_{2}-1\right)$ for $k=1,2, \ldots, n_{T}$ is the solution of (34), $u_{i, 0}^{k}=u_{i, N_{2}}^{k}=u_{0, j}^{k}=u_{N_{1}, j}^{k}=0$. Then, there exist positive constants $C_{1}$ independent of $n, \Delta x, \Delta y, \tau$ and $T$, and $C_{2}$ independent of $n, \Delta x, \Delta y$, and $\tau$, such that

$$
\left\|\mathbf { u } ^ { k } \left|\left\|_{l}^{2} \leq C_{1} \mid\right\| \mathbf{u}^{0}\left\|_{l}^{2}+C_{2} \max _{0 \leq t \leq T}\right\| \mathbf{f}(t) \|^{2}, \quad l=3 \text { or } 4\right.\right.
$$

where $\mathbf{u}^{k}, \mathbf{f}(t) \in \mathbb{R}^{\left(N_{1}+1\right) \times\left(N_{2}+1\right)}$ with $\left(\mathbf{u}^{k}\right)_{i, j}=u_{i, j}^{k}$ and $(\mathbf{f}(t))_{i, j}=f\left(x_{i}, y_{j}, t\right)$.

Theorem 3.4 (Convergence). Suppose that $U$ and $u_{i, j}^{n}\left(0 \leq i \leq N_{1}, 0 \leq j \leq N_{2}, 1 \leq n \leq n_{T}\right)$ are the solutions to (1) and (34), respectively. If $U \in C^{2}\left(0, T ; C^{6}(\Omega)\right), f \in C\left(0, T ; C^{4}(\Omega)\right)$ and $\phi_{0} \in C(\bar{\Omega})$, then there exists a positive constant $C$ independent of $k, \Delta x, \Delta y$ and $\tau$, such that

$$
\left\|\mathbf{u}^{n}-\mathbf{U}\left(t_{n}\right)\right\| \leq C\left(\tau^{2}+\Delta x^{4}+\Delta y^{4}+\Delta x^{2} \Delta y^{2}\right) .
$$

\section{Extension to three-dimensional time-fractional subdiffusion}

In this section, we extend the fast solver to solve the three-dimensional time-fractional subdiffusion equation

$$
\left\{\begin{array}{l}
{ }_{C} D_{0, t}^{\beta} U=\mu\left(\partial_{x}^{2}+\partial_{y}^{2}+\partial_{z}^{2}\right) U+f(x, y, z, t), \quad(x, y, z, t) \in \Omega \times(0, T], T>0, \\
U(x, y, z, 0)=\phi_{0}(x, y), \quad x \in \Omega \\
U(x, y, z, t)=0, \quad(x, y, z, t) \in \partial \Omega \times(0, T],
\end{array}\right.
$$


where $0<\beta<1, \mu>0, \Omega=\left(x_{L}, x_{R}\right) \times\left(y_{L}, y_{R}\right) \times\left(z_{L}, z_{R}\right)$.

Denote by $\Delta x, \Delta y$, and $\Delta z$ the step sizes in $x, y$, and $z$ directions, respectively, where $\Delta x=\left(x_{R}-x_{L}\right) / N_{1}, \Delta y=\left(y_{R}-y_{L}\right) / N_{2}$, and $\Delta z=\left(z_{R}-z_{L}\right) / N_{3}, N_{1}, N_{2}$, and $N_{3}$ are positive integers. Define the grid points $\left(x_{i}, y_{j}, z_{k}\right)$ as $x_{i}=x_{L}+i \Delta x\left(i=0,1, \ldots, N_{1}\right)$, $y_{j}=y_{L}+j \Delta y\left(j=0,1, \ldots, N_{2}\right)$, and $z_{k}=z_{L}+k \Delta z\left(k=0,1, \ldots, N_{3}\right)$, respectively. For simplicity, we denote $U_{i, j, k}^{n}=U\left(x_{i}, y_{j}, z_{k}, t_{n}\right), \delta_{x}^{2} U_{i, j, k}^{n}=\left(U_{i+1, j}^{n}-2 U_{i, j, k}^{n}+U_{i-1, j, k}^{n}\right) / \Delta x^{2}, \delta_{y}^{2} U_{i, j, k}^{n}=$ $\left(U_{i, j+1, k}^{n}-2 U_{i, j, k}^{n}+U_{i, j-1, k}^{n}\right) / \Delta y^{2}$, and $\delta_{z}^{2} U_{i, j, k}^{n}=\left(U_{i, j, k+1}^{n}-2 U_{i, j, k}^{n}+U_{i, j, k-1}^{n}\right) / \Delta z^{2}$.

\subsection{Central difference in space}

With the same time discretization in (24) and the central difference for the space derivatives, we obtain the fully FDMs for (41):

- FDM II $(q)$ : Find $u_{i, j, k}^{n}\left(0<i<N_{1}, 0<j<N_{2}, 0<k<N_{3}\right)$ for $n=1,2, \ldots, n_{T}$, such that

$$
\begin{aligned}
D^{(n)} u_{i, j, k}= & \mu L_{q}^{(n)}\left(\delta_{x}^{2}+\delta_{y}^{2}+\delta_{z}^{2}\right) u_{i, j, k}+\mu B_{n}^{(q)}\left(\delta_{x}^{2}+\delta_{y}^{2}+\delta_{z}^{2}\right) u_{i, j, k}^{0} \\
& +\mu C_{n}^{(q)}\left(\delta_{x}^{2}+\delta_{y}^{2}+\delta_{z}^{2}\right)\left(u_{i, j, k}^{1}-u_{i, j, k}^{0}\right)+\frac{1}{\tau^{\beta}} F_{i, j, k}^{n},
\end{aligned}
$$

where $D^{(n)}, L_{q}^{(n)}, B_{n}^{(q)}, C_{n}^{(q)}$, and $F^{n}$ are defined by (10), (11), (6), (7), and (14), respectively. The initial and boundary conditions of the scheme (42) are given by

$$
\begin{aligned}
& u_{i, j, k}^{0}=\phi_{0}\left(x_{i}, y_{j}, z_{k}\right), \quad 0 \leq i \leq N_{1}, 0 \leq j \leq N_{2}, 0 \leq k \leq N_{3}, \\
& u_{i, j, k}^{n}=0, \quad i=0, N_{1}, \text { or } j=0, N_{2}, \text { or } k=0, N_{3}, \quad 1 \leq n \leq n_{T} .
\end{aligned}
$$

Similar to (24), we can prove that the two difference schemes (42)-(43) are also unconditionally stable and convergent of order two both in time and space, which is omitted here. We briefly describe how to extend the fast solver developed in the last section to solve (42)-(43).

Denote $\Delta_{1} u_{i, j, k}^{n}=u_{i+1, j, k}^{n}-2 u_{i, j, k}^{n}+u_{i-1, j, k}^{n}, \Delta_{2} u_{i, j, k}^{n}=u_{i, j+1, k}^{n}-2 u_{i, j, k}^{n}+u_{i, j-1, k}^{n}$, and $\Delta_{3} u_{i, j, k}^{n}=u_{i, j, k+1}^{n}-2 u_{i, j, k}^{n}+u_{i, j, k-1}^{n}$. Then, from (42), we have

$$
\begin{aligned}
\sum_{r=0}^{n} \omega_{r} u_{i, j, k}^{n-r}-b_{n} u_{i, j, k}^{0}= & \left(\mu_{x} \Delta_{1}+\mu_{y} \Delta_{2}+\mu_{z} \Delta_{3}\right)\left(\sum_{r=0}^{n} \theta_{r}^{(q)} u_{i, j, k}^{n-r}+B_{n}^{(q)} u_{i, j, k}^{0}\right. \\
& \left.+C_{n}^{(q)}\left(u_{i, j, k}^{1}-u_{i, j, k}^{0}\right)\right)+F_{i, j, k}^{n},
\end{aligned}
$$

where $\mu_{x}=\mu \tau^{\beta} / \Delta x^{2}, \mu_{y}=\mu \tau^{\beta} / \Delta y^{2}$, and $\mu_{z}=\mu \tau^{\beta} / \Delta z^{2}$.

For simplicity, we first consider the fast solver for the following type model equation

$$
u_{i, j, k}+\left(\mu_{x} \Delta_{1}+\mu_{y} \Delta_{2}+\mu_{z} \Delta_{3}\right) u_{i, j, k}=F_{i, j, k},
$$

where $1 \leq i \leq N_{1}-1,1 \leq j \leq N_{2}-1,1 \leq k \leq N_{3}-1$, and $u_{0, j, k}=u_{N_{1}, j, k}=u_{i, 0, k}=$ $u_{i, N_{2}, k}=u_{i, j, 0}=u_{i, j, N_{3}}=0$. For simplicity, we define $u_{*, j, k}=\left(u_{1, j, k}, u_{2, j, k}, \ldots, u_{N_{x}-1, j, k}\right)^{T}$. The symbols $u_{i, *, k}$ and $u_{i, j, *}$ are defined similarly.

Next, we illustrate how to obtain $u_{i, j, k}$ efficiently from (45). 
(I) For fixed $j, k$, set $u_{*, j, k}=Q^{(1)} \hat{u}_{*, j, k}$. Like (29), we can obtain from (45)

$$
\begin{aligned}
& \hat{u}_{*, j, k}+\mu_{x} \Lambda^{(1)} \hat{u}_{*, j, k}+\mu_{y}\left(\hat{u}_{*, j+1, k}-2 \hat{u}_{*, j, k}+\hat{u}_{*, j-1, k}\right)+\mu_{z}\left(\hat{u}_{*, j, k+1}-2 \hat{u}_{*, j, k}+\hat{u}_{*, j, k-1}\right) \\
= & 2 h_{1} Q^{(1)} F_{*, j, k}=2 h_{1} \hat{F}_{*, j, k} .
\end{aligned}
$$

The above equation (46) implies

$$
\left(1+\mu_{x} \lambda_{i}^{(1)}\right) \hat{u}_{i, j, k}+\left(\mu_{y} \Delta_{2}+\mu_{z} \Delta_{3}\right) \hat{u}_{i, j, k}=2 h_{1} \hat{F}_{i, j, k}
$$

(II) For fixed $i, k$, set $\hat{u}_{i, *, k}=Q^{(2)} \hat{\hat{u}}_{i, *, k}$. Similar to (46), we can derive from (47) that

$$
\hat{\hat{u}}_{i, *, k}+\mu_{x} \lambda_{i}^{(1)} \hat{\hat{u}}_{i, *, k}+\mu_{y} \Lambda^{(2)} \hat{\hat{u}}_{i, *, k}+\mu_{z}\left(\hat{\hat{u}}_{i, *, k+1}-2 \hat{\hat{u}}_{i, *, k}+\hat{\hat{u}}_{i, *, k-1}\right)=4 h_{1} h_{2} Q^{(2)} \hat{F}_{i, *, k} .
$$

Let $=\hat{\hat{F}}_{i, *, k}=Q^{(2)} \hat{F}_{i, *, k}$. Then we have from (48) that

$$
\left(1+\mu_{x} \lambda_{i}^{(1)}+\mu_{y} \lambda_{j}^{(2)}\right) \hat{\hat{u}}_{i, j, k}+\mu_{z} \Delta_{3} \hat{\hat{u}}_{i, j, k}=4 h_{1} h_{2} \hat{\hat{F}}_{i, j, k}
$$

(III) For fixed $i, j$, set $\hat{\hat{u}}_{i, j, *}=Q^{(3)} v_{i, j, *}$, we can derive from (49) that

$$
v_{i, j, *}+\mu_{x} \lambda_{i}^{(1)} v_{i, j, *}+\mu_{y} \lambda_{j}^{(2)} v_{i, j, *}+\mu_{z} \Lambda^{(3)} v_{i, j, *}=8 h_{1} h_{2} h_{3} Q^{(3)} \hat{\hat{F}}_{i, j, *}
$$

Let $G_{i, j, *}=Q^{(3)} \hat{\hat{F}}_{i, j, *}$. Then we derive from (50) that

$$
v_{i, j, k}+\mu_{x} \lambda_{i}^{(1)} v_{i, j, k}+\mu_{y} \lambda_{j}^{(2)} v_{i, j, k}+\mu_{z} \lambda_{k}^{(3)} v_{i, j, k}=8 h_{1} h_{2} h_{3} G_{i, j, k}
$$

which yields $v_{i, j, k}=\frac{8 h_{1} h_{2} h_{3} G_{i, j, k}}{1+\mu_{x} \lambda_{i}^{(1)}+\mu_{y} \lambda_{j}^{(2)}+\mu_{z} \lambda_{k}^{(3)}}$. Hence, we can recover $u_{i, j, k}$ by the following formulas

$$
u_{*, j, k}=Q^{(1)} \hat{u}_{*, j, k}, \quad \hat{u}_{i, *, k}=Q^{(2)} \hat{\hat{u}}_{i, *, k}, \quad \hat{\hat{u}}_{i, j, *}=Q^{(3)} v_{i, j, *} .
$$

In the above steps (I)-(III), all the matrix-vector products can be implemented using the fast Fourier transform (FFT). One can obtain that the equation (45) can be solved with $O\left(N^{3} \log (N)\right)$ operations, where $N=\max \left\{N_{1}, N_{2}, N_{3}\right\}$.

We now describe how to compute $u_{i, j, k}^{n}$ in (44) with linearithmic complexity:

1) For $j=1,2, \ldots, N_{2}-1$ and $k=1,2, \ldots, N_{3}-1$, compute $\hat{F}_{*, j, k}=Q^{(1)} F_{*, j, k}^{n}$ with FFT;

2) For $i=1,2, \ldots, N_{1}-1$ and $k=1,2, \ldots, N_{3}-1$, compute $\hat{\hat{F}}_{i, *, k}=Q^{(2)} \hat{F}_{i, *, k}$ with FFT;

3) For $i=1,2, \ldots, N_{1}-1$ and $j=1,2, \ldots, N_{2}-1$, compute $G_{i, j, *}^{n}=Q^{(3)} \hat{F}_{i, j, *}$ with FFT; 
4) Compute $v_{i, j, k}^{n}$ from the following equation

$$
\begin{aligned}
\sum_{r=0}^{n} \omega_{r}\left(v_{i, j, k}^{n-r}-v_{i, j, k}^{0}\right)= & -\left(\mu_{x} \lambda_{i}^{(1)}+\mu_{y} \lambda_{j}^{(2)}+\mu_{z} \lambda_{k}^{(3)}\right)\left[\sum_{r=0}^{n} \theta_{r}^{(q)} v_{i, j, k}^{n-r}\right. \\
& \left.+B_{n}^{(q)} v_{i, j, k}^{0}+C_{n}^{(q)}\left(v_{i, j, k}^{1}-v_{i, j, k}^{0}\right)\right]+8 h_{1} h_{2} h_{3} G_{i, j, k}^{n}
\end{aligned}
$$

5) For $i=1,2, \ldots, N_{1}-1$ and $j=1,2, \ldots, N_{2}-1$, compute $\hat{\hat{u}}_{i, j, *}=Q^{(3)} v_{i, j, *}^{n}$ with FFT;

6) For $i=1,2, \ldots, N_{1}-1$ and $k=1,2, \ldots, N_{3}-1$, compute $\hat{u}_{i, *, k}=Q^{(2)} \hat{\hat{u}}_{i, *, k}$ with FFT;

7) For $j=1,2, \ldots, N_{2}-1$ and $k=1,2, \ldots, N_{3}-1$, compute $u_{*, j, k}^{n}=Q^{(1)} \hat{u}_{*, j, k}$ with FFT.

\subsection{Compact difference in space}

In this section, we develop the fast compact difference schemes for three-dimensional time-fractional subdiffusion equation (41). Similar to (33), we can obtain the following approach

$$
\widetilde{\mathcal{H}} U_{i, j, k}=\widetilde{\mathcal{A}} f_{i, j, k}+O\left(\Delta x^{4}+\Delta y^{4}+\Delta z^{4}+\Delta x^{2} \Delta y^{2}+\Delta x^{2} \Delta z^{2}+\Delta y^{2} \Delta z^{2}\right)
$$

for the model problem $\left(\partial_{x}^{2}+\partial_{y}^{2}+\partial_{z}^{2}\right) U=f(x, y, z)$ with homogeneous boundary conditions, where $0<i<N_{1}, 0<j<N_{2}, 0<k<N_{3}, \widetilde{\mathcal{A}}$ and $\widetilde{\mathcal{H}}$ are respectively defined by

$$
\begin{aligned}
\widetilde{\mathcal{A}} U_{i, j, k}= & U_{i, j, k}+\frac{1}{12}\left(\Delta x^{2} \delta_{x}^{2}+\Delta y^{2} \delta_{y}^{2}+\Delta z^{2} \delta_{z}^{2}\right) U_{i, j, k} \\
\widetilde{\mathcal{H}} U_{i, j, k}= & \left(\delta_{x}^{2}+\delta_{y}^{2}+\delta_{z}^{2}\right) U_{i, j, k}+\frac{1}{12}\left[\left(\Delta x^{2}+\Delta y^{2}\right) \delta_{x}^{2} \delta_{y}^{2}+\left(\Delta x^{2}+\Delta z^{2}\right) \delta_{x}^{2} \delta_{z}^{2}\right. \\
& \left.+\left(\Delta y^{2}+\Delta z^{2}\right) \delta_{y}^{2} \delta_{z}^{2}\right] U_{i, j, k} .
\end{aligned}
$$

Similar to (34), we obtain the compact finite difference methods for (41) from (53):

- CFDM II $(q)$ : Find $u_{i, j, k}^{n}\left(0<i<N_{1}, 0<j<N_{2}, 0<k<N_{3}\right)$ for $n=1,2, \ldots, n_{T}$, such that

$$
D^{(n)} \widetilde{\mathcal{A}} u_{i, j, k}=\mu L_{q}^{(n)} \widetilde{\mathcal{H}} u_{i, j, k}+\mu B_{n}^{(q)} \widetilde{\mathcal{H}} u_{i, j, k}^{0}+\mu C_{n}^{(q)}\left(\widetilde{\mathcal{H}} u_{i, j, k}^{1}-\widetilde{\mathcal{H}} u_{i, j, k}^{0}\right)+\frac{1}{\tau^{\beta}} \widetilde{\mathcal{A}} F_{i, j, k}^{n},
$$

where $D^{(n)}, L_{q}^{(n)}, B_{n}^{(q)}, C_{n}^{(q)}$ and $F^{n}$, are defined by (10), (11), (6), (7), and (14), respectively. The initial and boundary conditions for (54) are as in (43).

We can also prove that the two compact difference schemes (54) are unconditionally stable with convergence order of two in time and four in space. 
We can extend the fast solver for (42) to (54) by simply replacing (52) with the following

$$
\begin{aligned}
& {\left[1-\left(\lambda_{i}^{(1)}+\lambda_{j}^{(2)}+\lambda_{k}^{(3)}\right) / 12\right] \sum_{r=0}^{n} \omega_{r}\left(v_{i, j, k}^{n-r}-v_{i, j, k}^{0}\right) } \\
= & -\left(\mu_{x} \lambda_{i}^{(1)}+\mu_{y} \lambda_{j}^{(2)}+\mu_{z} \lambda_{k}^{(3)}-\mu_{x y} \lambda_{i}^{(1)} \lambda_{j}^{(2)}-\mu_{x z} \lambda_{i}^{(1)} \lambda_{k}^{(3)}-\mu_{y z} \lambda_{j}^{(2)} \lambda_{k}^{(3)}\right) \\
& \times\left[\sum_{r=0}^{n} \theta_{r}^{(q)} v_{i, j, k}^{n-r}+B_{n}^{(q)} v_{i, j, k}^{0}+C_{n}^{(q)}\left(v_{i, j, k}^{1}-v_{i, j, k}^{0}\right)\right]+8 h_{1} h_{2} h_{3} G_{i, j, k}^{n}\left[1-\left(\lambda_{i}^{(1)}+\lambda_{j}^{(2)}+\lambda_{k}^{(3)}\right) / 12\right],
\end{aligned}
$$

where $\mu_{x y}=\frac{\mu \tau^{\beta}\left(\Delta x^{2}+\Delta y^{2}\right)}{12 \Delta x^{2} \Delta y^{2}}, \mu_{x z}=\frac{\mu \tau^{\beta}\left(\Delta x^{2}+\Delta z^{2}\right)}{12 \Delta x^{2} \Delta z^{2}}$, and $\mu_{y z}=\frac{\mu \tau^{\beta}\left(\Delta y^{2}+\Delta z^{2}\right)}{12 \Delta y^{2} \Delta z^{2}}$.

Remark 4.1. The above fast solver for solving (54) has the same complexity $O\left(N^{3} \log (N)\right)$ as that for (42). We can also establish corresponding FDMs and compact FDMs to solve d-dimensional time-fractional PDEs with the corresponding fast solvers designed, the computational complexity of which in space is $O\left(N^{d} \log (N)\right)$.

\section{Numerical examples}

In this section, we present several numerical examples to verify the error estimates and the convergence orders of the proposed ADI methods and non-ADI methods. We also compare our proposed methods with some existing ADI methods. Our programs are written in Matlab codes, which were run in a 64 bit Windows 7 laptop with a $2.50 \mathrm{GHz} \mathrm{CPU}$ and a $4 \mathrm{~GB}$ RAM.

Example 5.1. Consider the following time-fractional subdiffusion equation

$$
\left\{\begin{array}{l}
{ }_{C} D_{0, t}^{\beta} U=\left(\partial_{x}^{2}+\partial_{y}^{2}\right) U+f(x, y, t), \quad(x, y, t) \in \Omega \times(0,1], \\
U(x, y, 0)=\sin (p(x+y)), \quad(x, y) \in \bar{\Omega},
\end{array}\right.
$$

where $\Omega=(0,1) \times(0,1)$ and the function $f$ is chosen such that the solution to (56) is

$$
U(x, y, t)=\left(t^{2+\beta}+t+1\right) \sin (p(x+y)) .
$$

Denote $\left(\mathbf{e}^{n}(\tau, \Delta x, \Delta y)\right)_{i, j}=e_{i, j}^{n}=U\left(x_{i}, y_{j}, t_{n}\right)-u_{i, j}^{n}$ as the error at time level $n$. Then the convergence order in time at $t=1\left(n=n_{T}\right)$ is given by

$$
\text { order }=\frac{\log \left(\left\|e^{n}\left(\tau_{1}, \Delta x, \Delta y\right)\right\| /\left\|e^{n}\left(\tau_{2}, \Delta x, \Delta y\right)\right\|\right)}{\log \left(\tau_{1} / \tau_{2}\right)},
$$

where $\tau_{1}$ and $\tau_{2}$ are time step sizes and $\tau_{1} \neq \tau_{2}$. In Table 1 , we present the $L^{2}$ errors $\left\|\mathbf{e}^{n}\right\|$ at $t=1\left(n=n_{T}\right)$ for different schemes. For different fractional orders $\beta=0.2,0.5,0.8$, FDM I and CFDM I show second-order accuracy in time, and ADI-FDMs are $(1+\beta)$-order accurate in time, which are in agreement with the theoretical analysis. In Table 2 we observe that the convergence rate in space for FDM I is second-order and for CFDM I the convergence rate is fourth-order as expected from our theoretical analysis. We also find from Table 1 that the 
Table 1: The $L^{2}$ errors at $t=1$ for Example 5.1, $N_{1}=N_{2}=500, p=1$.

\begin{tabular}{c|c|cc|cc|cc}
\hline Methods & $1 / \tau$ & $\beta=0.2$ & order & $\beta=0.5$ & order & $\beta=0.8$ & order \\
\hline \multirow{3}{*}{ ADI FDM (1) } & 8 & $4.4733 \mathrm{e}-3$ & & $1.8479 \mathrm{e}-3$ & & $5.5596 \mathrm{e}-4$ & \\
$(17)$ & 16 & $2.3808 \mathrm{e}-3$ & 0.91 & $7.5043 \mathrm{e}-4$ & 1.30 & $1.8228 \mathrm{e}-4$ & 1.61 \\
& 32 & $1.1230 \mathrm{e}-3$ & 1.08 & $2.8193 \mathrm{e}-4$ & 1.41 & $5.6286 \mathrm{e}-5$ & 1.70 \\
& 64 & $5.0625 \mathrm{e}-4$ & 1.15 & $1.0268 \mathrm{e}-4$ & 1.46 & $1.6953 \mathrm{e}-5$ & 1.73 \\
\hline \multirow{3}{*}{ ADI FDM (2) } & 8 & $4.7329 \mathrm{e}-3$ & & $2.1800 \mathrm{e}-3$ & & $7.2306 \mathrm{e}-4$ & \\
$(17)$ & 16 & $2.5381 \mathrm{e}-3$ & 0.90 & $8.7515 \mathrm{e}-4$ & 1.32 & $2.2983 \mathrm{e}-4$ & 1.65 \\
& 32 & $1.1999 \mathrm{e}-3$ & 1.08 & $3.2564 \mathrm{e}-4$ & 1.43 & $6.9325 \mathrm{e}-5$ & 1.73 \\
& 64 & $5.4122 \mathrm{e}-4$ & 1.15 & $1.1773 \mathrm{e}-4$ & 1.47 & $2.0497 \mathrm{e}-5$ & 1.76 \\
\hline \multirow{3}{*}{ FDM I $(1)$} & 8 & $9.1153 \mathrm{e}-5$ & & $2.3356 \mathrm{e}-4$ & & $3.0418 \mathrm{e}-4$ & \\
$(24)$ & 16 & $2.3758 \mathrm{e}-5$ & 1.94 & $5.9100 \mathrm{e}-5$ & 1.98 & $7.6522 \mathrm{e}-5$ & 1.99 \\
& 32 & $6.0634 \mathrm{e}-6$ & 1.97 & $1.4894 \mathrm{e}-5$ & 1.99 & $1.9269 \mathrm{e}-5$ & 1.99 \\
& 64 & $1.5697 \mathrm{e}-6$ & 1.95 & $3.7816 \mathrm{e}-6$ & 1.98 & $4.8749 \mathrm{e}-6$ & 1.98 \\
\hline \multirow{3}{*}{ FDM I $(2)$} & 8 & $3.4215 \mathrm{e}-5$ & & $1.1385 \mathrm{e}-4$ & & $2.0571 \mathrm{e}-4$ & \\
$(24)$ & 16 & $1.0119 \mathrm{e}-5$ & 1.76 & $2.9953 \mathrm{e}-5$ & 1.92 & $5.2029 \mathrm{e}-5$ & 1.98 \\
& 32 & $2.7267 \mathrm{e}-6$ & 1.89 & $7.6478 \mathrm{e}-6$ & 1.97 & $1.3093 \mathrm{e}-5$ & 1.99 \\
& 64 & $7.4401 \mathrm{e}-7$ & 1.87 & $1.9683 \mathrm{e}-6$ & 1.96 & $3.3230 \mathrm{e}-6$ & 1.98 \\
\hline \multirow{3}{*}{ CFDM I (1) } & 8 & $9.1090 \mathrm{e}-5$ & & $2.3349 \mathrm{e}-4$ & & $3.0411 \mathrm{e}-4$ & \\
$(34)$ & 16 & $2.3695 \mathrm{e}-5$ & 1.94 & $5.9036 \mathrm{e}-5$ & 1.98 & $7.6459 \mathrm{e}-5$ & 1.99 \\
& 32 & $6.0002 \mathrm{e}-6$ & 1.98 & $1.4831 \mathrm{e}-5$ & 1.99 & $1.9206 \mathrm{e}-5$ & 1.99 \\
& 64 & $1.5065 \mathrm{e}-6$ & 1.99 & $3.7187 \mathrm{e}-6$ & 2.00 & $4.8123 \mathrm{e}-6$ & 2.00 \\
\hline \multirow{2}{*}{ CFDM I (2) } & 8 & $3.4152 \mathrm{e}-5$ & & $1.1379 \mathrm{e}-4$ & & $2.0565 \mathrm{e}-4$ & \\
$(34)$ & 16 & $1.0056 \mathrm{e}-5$ & 1.76 & $2.9890 \mathrm{e}-5$ & 1.93 & $5.1967 \mathrm{e}-5$ & 1.98 \\
& 32 & $2.6635 \mathrm{e}-6$ & 1.92 & $7.5849 \mathrm{e}-6$ & 1.98 & $1.3030 \mathrm{e}-5$ & 2.00 \\
& 64 & $6.8087 \mathrm{e}-7$ & 1.97 & $1.9054 \mathrm{e}-6$ & 1.99 & $3.2604 \mathrm{e}-6$ & 2.00 \\
\hline
\end{tabular}

Table 2: The $L^{2}$ errors at $t=1$ for Example 5.1, $\beta=0.5, p=2 \pi, \tau=10^{-3}$.

\begin{tabular}{cc|cc|cc}
\hline$N_{1}$ & $N_{2}$ & FDM I (1) & order & FDM I (2) & order \\
\hline 8 & 8 & $9.2764 \mathrm{e}-2$ & & $9.2763 \mathrm{e}-2$ & \\
16 & 16 & $2.2491 \mathrm{e}-2$ & 2.04 & $2.2491 \mathrm{e}-2$ & 2.04 \\
32 & 32 & $5.5780 \mathrm{e}-3$ & 2.01 & $5.5779 \mathrm{e}-3$ & 2.01 \\
64 & 64 & $1.3917 \mathrm{e}-3$ & 2.00 & $1.3916 \mathrm{e}-3$ & 2.00 \\
\hline \hline$N_{1}$ & $N_{2}$ & CFDM I (1) & order & CFDM I (2) & order \\
\hline 4 & 4 & $2.1670 \mathrm{e}-2$ & & $2.1670 \mathrm{e}-2$ & \\
6 & 6 & $5.2694 \mathrm{e}-3$ & 3.49 & $5.2695 \mathrm{e}-3$ & 3.49 \\
8 & 8 & $1.7489 \mathrm{e}-3$ & 3.83 & $1.7489 \mathrm{e}-3$ & 3.83 \\
10 & 10 & $7.2956 \mathrm{e}-4$ & 3.92 & $7.2963 \mathrm{e}-4$ & 3.92 \\
\hline
\end{tabular}

non-ADI methods show better performance over the ADI methods due to the higher-order accuracy in time of the non-ADI methods, see also Tables 3 and 4.

In Tables 3 and 4, the four non-ADI methods show much better performance over the two 
ADI methods. This can be explained as follows: first, the non-ADI methods have higherorder accuracy than the ADI methods in time; second, the perturbation terms introduced in the ADI methods dominate the accuracy. In this example, we have $\tau^{\beta} \partial_{x}^{2} \partial_{y}^{2} U^{n}=\tau^{\beta} p^{4}\left(t_{n}^{2+\beta}+\right.$ $\left.t_{n}+1\right) \sin (p(x+y))$, and the perturbation term $\tau^{\beta} \partial_{x}^{2} \partial_{y}^{2}\left(U^{n}-U^{n-1}\right)$ in (15) increases when $p$ increases and/or $\beta$ decreases. As this term dominates the total accuracy of the ADI methods, we observe that numerical solutions of the ADI methods are less accurate when $p$ increases and/or $\beta$ decreases, see Table $1(p=1)$, Table $3(p=2 \pi)$, and Table $4(p=4 \pi)$.

Table 3: The $L^{2}$ errors at $t=1$ for Example 5.1, $N_{1}=N_{2}=500, p=2 \pi$.

\begin{tabular}{c|c|c|c|c|c}
\hline Methods & $1 / \tau$ & $\beta=0.1$ & $\beta=0.2$ & $\beta=0.65$ & $\beta=0.8$ \\
\hline \multirow{3}{*}{ ADI FDM (1) } & 8 & $1.3688 \mathrm{e}-0$ & $1.1769 \mathrm{e}-0$ & $4.3274 \mathrm{e}-1$ & $2.8822 \mathrm{e}-1$ \\
$(17)$ & 16 & $9.4404 \mathrm{e}-1$ & $7.1764 \mathrm{e}-1$ & $1.5975 \mathrm{e}-1$ & $9.1831 \mathrm{e}-2$ \\
& 32 & $5.4659 \mathrm{e}-1$ & $3.7177 \mathrm{e}-1$ & $5.3707 \mathrm{e}-2$ & $2.7276 \mathrm{e}-2$ \\
& 64 & $2.8630 \mathrm{e}-1$ & $1.7607 \mathrm{e}-1$ & $1.7428 \mathrm{e}-2$ & $7.9047 \mathrm{e}-3$ \\
\hline \multirow{3}{*}{ FDM I (1) } & 8 & $2.4643 \mathrm{e}-4$ & $4.9859 \mathrm{e}-4$ & $1.1639 \mathrm{e}-3$ & $9.5418 \mathrm{e}-4$ \\
(24) & 16 & $8.1543 \mathrm{e}-5$ & $1.4510 \mathrm{e}-4$ & $3.1047 \mathrm{e}-4$ & $2.5837 \mathrm{e}-4$ \\
& 32 & $3.7749 \mathrm{e}-5$ & $5.3530 \mathrm{e}-5$ & $9.4946 \mathrm{e}-5$ & $8.2104 \mathrm{e}-5$ \\
& 64 & $2.6577 \mathrm{e}-5$ & $3.0499 \mathrm{e}-5$ & $4.0868 \mathrm{e}-5$ & $3.7658 \mathrm{e}-5$ \\
\hline \multirow{3}{*}{ CFDM I (1) } & 8 & $2.2348 \mathrm{e}-4$ & $4.7549 \mathrm{e}-4$ & $1.1398 \mathrm{e}-3$ & $9.3015 \mathrm{e}-4$ \\
(34) & 16 & $5.8667 \mathrm{e}-5$ & $1.2215 \mathrm{e}-4$ & $2.8728 \mathrm{e}-4$ & $2.3542 \mathrm{e}-4$ \\
& 32 & $1.4903 \mathrm{e}-5$ & $3.0654 \mathrm{e}-5$ & $7.2089 \mathrm{e}-5$ & $5.9339 \mathrm{e}-5$ \\
& 64 & $3.7446 \mathrm{e}-6$ & $7.6540 \mathrm{e}-6$ & $1.8086 \mathrm{e}-5$ & $1.4906 \mathrm{e}-5$ \\
\hline
\end{tabular}

Table 4: The $L^{2}$ errors at $t=1$ for Example 5.1, $N_{1}=N_{2}=500, p=4 \pi$.

\begin{tabular}{|c|c|c|c|c|c|}
\hline Methods & $1 / \tau$ & $\beta=0.1$ & $\beta=0.2$ & $\beta=0.9$ & $\beta=1$ \\
\hline \multirow{4}{*}{$\begin{array}{l}\text { ADI FDM (2) } \\
(17)\end{array}$} & 8 & $1.7455 \mathrm{e}-0$ & $1.6371 \mathrm{e}-0$ & $6.9989 \mathrm{e}-1$ & $5.5231 \mathrm{e}-1$ \\
\hline & 16 & $1.5015 \mathrm{e}-0$ & $1.3438 \mathrm{e}-0$ & $2.6548 \mathrm{e}-1$ & $1.7964 \mathrm{e}-1$ \\
\hline & 32 & $1.1930 \mathrm{e}-0$ & $1.0004 \mathrm{e}-0$ & 7.9343e-2 & $4.8354 \mathrm{e}-2$ \\
\hline & 64 & $8.6097 \mathrm{e}-1$ & $6.3708 \mathrm{e}-1$ & $2.1867 \mathrm{e}-2$ & $1.2251 \mathrm{e}-2$ \\
\hline \multirow{4}{*}{$\begin{array}{l}\text { FDM I }(2) \\
\quad(24)\end{array}$} & 8 & $5.6337 \mathrm{e}-5$ & $4.3830 \mathrm{e}-5$ & $1.0623 \mathrm{e}-4$ & $1.4362 \mathrm{e}-4$ \\
\hline & 16 & $9.7399 \mathrm{e}-5$ & $9.7110 \mathrm{e}-5$ & $1.0565 \mathrm{e}-4$ & $1.0807 \mathrm{e}-4$ \\
\hline & 32 & $1.0161 \mathrm{e}-4$ & $1.0170 \mathrm{e}-4$ & $1.0286 \mathrm{e}-4$ & $1.0294 \mathrm{e}-4$ \\
\hline & 64 & $1.0201 \mathrm{e}-4$ & $1.0203 \mathrm{e}-4$ & $1.0218 \mathrm{e}-4$ & $1.0218 \mathrm{e}-4$ \\
\hline \multirow{4}{*}{$\begin{array}{c}\text { CFDM I (2) } \\
(34)\end{array}$} & 8 & $4.5841 \mathrm{e}-5$ & $5.8381 \mathrm{e}-5$ & $2.2204 \mathrm{e}-6$ & $2.3624 \mathrm{e}-5$ \\
\hline & 16 & $4.7025 \mathrm{e}-6$ & $4.9968 \mathrm{e}-6$ & $3.4970 \mathrm{e}-6$ & $4.2238 \mathrm{e}-6$ \\
\hline & 32 & $4.6017 \mathrm{e}-7$ & $3.6081 \mathrm{e}-7$ & $9.1015 \mathrm{e}-7$ & $1.0007 \mathrm{e}-6$ \\
\hline & 64 & $3.9600 \mathrm{e}-8$ & $8.3870 \mathrm{e}-9$ & $2.2672 \mathrm{e}-7$ & $2.4847 \mathrm{e}-7$ \\
\hline
\end{tabular}

In Table 5, we compare the CPU time of the ADI methods, the fast solvers, and the direct solvers. We can see that the ADI methods are most efficient, while the direct solvers are most costly, i.e., the direct solvers need more time and memory storage, see the last 
column in Table 5, in which "out of memory" error occurred when using a laptop with 4G memory. The fast solver performs well, which is much less costly than the direct solver.

Table 5: Comparison of CPU time (s) for different methods and solvers at $t=1, \beta=0.5, N_{1}=N_{2}=2^{k}, p=$ $2 \pi, \tau=1 / 4$.

\begin{tabular}{l|cc|cc}
\hline Methods & $k=10$ & cputime $(\mathrm{s})$ & $k=11$ & cputime (s) \\
\hline ADI FDM (1) & $6.4395 \mathrm{e}-1$ & 3.8410 & $6.4396 \mathrm{e}-1$ & 19.1250 \\
ADI FDM (2) & $6.6312 \mathrm{e}-1$ & 4.1191 & $6.6313 \mathrm{e}-1$ & 18.8665 \\
\hline FDM I (1) with fast solver & $3.4119 \mathrm{e}-3$ & 4.3650 & $3.4081 \mathrm{e}-3$ & 21.7719 \\
FDM I (2) with fast solver & $4.3155 \mathrm{e}-4$ & 4.4421 & $4.3509 \mathrm{e}-4$ & 21.6415 \\
\hline FDM (1) with direct solver & $3.4119 \mathrm{e}-3$ & 19.3573 & - & out of \\
FDM (2) with direct solver & $4.3155 \mathrm{e}-4$ & 18.0814 & - & memory \\
\hline CFDM (1) with fast solver & $3.4068 \mathrm{e}-3$ & 4.9503 & $3.4068 \mathrm{e}-3$ & 24.3992 \\
CFDM (2) with fast solver & $4.3627 \mathrm{e}-4$ & 4.9033 & $4.3627 \mathrm{e}-4$ & 23.5919 \\
\hline CFDM (1) with direct solver & $3.4068 \mathrm{e}-3$ & 30.9193 & - & out of \\
CFDM (2) with direct solver & $4.3627 \mathrm{e}-4$ & 29.8666 & - & memory \\
\hline
\end{tabular}

Example 5.2. Consider the following subdiffusion equation [3]

$$
\left\{\begin{array}{l}
{ }_{C} D_{0, t}^{\beta} U=\left(\partial_{x}^{2}+\partial_{y}^{2}\right) U+f(x, y, t), \quad(x, y, t) \in \Omega \times(0,1 / 2] \\
U(x, y, 0)=0, \quad(x, y) \in \bar{\Omega} \\
U(x, y, t)=0, \quad(x, y, t) \in \partial \Omega \times(0,1 / 2]
\end{array}\right.
$$

where $0<\beta<1, \Omega=(0, \pi) \times(0, \pi)$, and

$$
f(x, t)=\left(\frac{2}{\Gamma(3-\beta)} t^{2-\beta}-2 t^{2}\right) \sin (x) \sin (y) .
$$

The exact solution of $(57)$ is $U(x, y, t)=t^{2} \sin (x) \sin (y)$.

In this example, we compare the proposed six methods with two ADI methods developed in [3]: the L1-ADI method with convergence rate $O\left(\Delta x^{2}+\Delta y^{2}+\tau^{\min \{2 \beta, 2-\beta\}}\right)$ and BD-ADI method with convergence rate $O\left(\Delta x^{2}+\Delta y^{2}+\tau^{\min \{1+\beta, 2-\beta\}}\right)$. In Table 6 , we check the maximum- $L^{\infty}$ error $\max _{0 \leq n \leq n_{T}}\left\|\mathbf{e}^{n}\right\|_{\infty}$, where

$$
\left\|\mathbf{e}^{n}\right\|_{\infty}=\left\|\mathbf{u}^{n}-\mathbf{U}\left(t_{n}\right)\right\|_{\infty}=\max _{0 \leq i \leq N_{1}} \max _{0 \leq j \leq N_{2}}\left|U\left(x_{i}, y_{j}, t_{n}\right)-u_{i, j}^{n}\right|
$$

From Table 6, we find that our six methods outperform both the L1-ADI method and the $\mathrm{BD}$-ADI method when $\beta>1 / 2$ and get almost similar results when $\beta \leq 1 / 2$ for the present ADI methods as the theoretical predictions suggest. 
Table 6: The maximum $L^{\infty}$ error for Example 2, $N_{1}=N_{2}=400, T=1 / 2$.

\begin{tabular}{l|c|c|c|c|c}
\hline Methods & $\beta$ & $1 / \tau=20$ & $1 / \tau=40$ & $1 / \tau=80$ & $1 / \tau=160$ \\
\hline FDM I (1) (24) & & $1.6176 \mathrm{e}-4$ & $4.3752 \mathrm{e}-5$ & $1.1833 \mathrm{e}-5$ & $3.5286 \mathrm{e}-6$ \\
FDM I (2) (24) & & $6.7232 \mathrm{e}-5$ & $2.0571 \mathrm{e}-5$ & $6.0693 \mathrm{e}-6$ & $2.0894 \mathrm{e}-6$ \\
CFDM I (1) (34) & & $1.6107 \mathrm{e}-4$ & $4.3059 \mathrm{e}-5$ & $1.1167 \mathrm{e}-5$ & $2.8893 \mathrm{e}-6$ \\
CFDM I (2) (34) & $1 / 3$ & $6.6539 \mathrm{e}-5$ & $2.0159 \mathrm{e}-5$ & $5.8820 \mathrm{e}-6$ & $1.6879 \mathrm{e}-6$ \\
ADI FDM (1) (17) & & $3.0166 \mathrm{e}-3$ & $1.2591 \mathrm{e}-3$ & $5.1248 \mathrm{e}-4$ & $2.0587 \mathrm{e}-4$ \\
ADI FDM (2) (17) & & $3.4357 \mathrm{e}-3$ & $1.4155 \mathrm{e}-3$ & $5.7189 \mathrm{e}-4$ & $2.2874 \mathrm{e}-4$ \\
BD-ADI [3] & & $3.8684 \mathrm{e}-3$ & $1.6437 \mathrm{e}-3$ & $6.7951 \mathrm{e}-4$ & $2.7672 \mathrm{e}-4$ \\
\hline FDM I (1) (24) & & $1.8818 \mathrm{e}-4$ & $5.1361 \mathrm{e}-5$ & $1.3845 \mathrm{e}-5$ & $3.9955 \mathrm{e}-6$ \\
FDM I (2) (24) & & $8.5033 \mathrm{e}-5$ & $2.6901 \mathrm{e}-5$ & $8.1907 \mathrm{e}-6$ & $2.4134 \mathrm{e}-6$ \\
CFDM I (1) (34) & & $1.8758 \mathrm{e}-4$ & $5.0757 \mathrm{e}-5$ & $1.3402 \mathrm{e}-5$ & $3.5394 \mathrm{e}-6$ \\
CFDM I (2) (34) & & $8.4428 \mathrm{e}-5$ & $2.6760 \mathrm{e}-5$ & $8.1531 \mathrm{e}-6$ & $2.4026 \mathrm{e}-6$ \\
ADI FDM (1) (17) & $1 / 2$ & $1.2064 \mathrm{e}-3$ & $4.6109 \mathrm{e}-4$ & $1.7027 \mathrm{e}-4$ & $6.1562 \mathrm{e}-5$ \\
ADI FDM (2) (17) & & $1.4882 \mathrm{e}-3$ & $5.5154 \mathrm{e}-4$ & $1.9984 \mathrm{e}-4$ & $7.1389 \mathrm{e}-5$ \\
L1-ADI [3] & & $3.4910 \mathrm{e}-3$ & $1.9907 \mathrm{e}-3$ & $1.0823 \mathrm{e}-3$ & $5.7133 \mathrm{e}-4$ \\
BD-ADI [3] & & $8.8067 \mathrm{e}-4$ & $3.2549 \mathrm{e}-4$ & $1.1618 \mathrm{e}-4$ & $4.0678 \mathrm{e}-5$ \\
\hline FDM I (1) (24) & & $1.7513 \mathrm{e}-4$ & $4.8460 \mathrm{e}-5$ & $1.3182 \mathrm{e}-5$ & $3.7961 \mathrm{e}-6$ \\
FDM I (2) (24) & & $8.6358 \mathrm{e}-5$ & $2.8351 \mathrm{e}-5$ & $8.7942 \mathrm{e}-6$ & $2.6181 \mathrm{e}-6$ \\
CFDM I (1) (34) & & $1.7461 \mathrm{e}-4$ & $4.7957 \mathrm{e}-5$ & $1.2938 \mathrm{e}-5$ & $3.4862 \mathrm{e}-6$ \\
CFDM I (2) (34) & & $8.6058 \mathrm{e}-5$ & $2.8257 \mathrm{e}-5$ & $8.7674 \mathrm{e}-6$ & $2.6073 \mathrm{e}-6$ \\
ADI FDM (1) (17) & $2 / 3$ & $4.0862 \mathrm{e}-4$ & $1.4601 \mathrm{e}-4$ & $4.9651 \mathrm{e}-5$ & $1.6243 \mathrm{e}-5$ \\
ADI FDM (2) (17) & & $5.7409 \mathrm{e}-4$ & $1.9296 \mathrm{e}-4$ & $6.3057 \mathrm{e}-5$ & $2.0108 \mathrm{e}-5$ \\
L1-ADI [3] & & $1.4331 \mathrm{e}-3$ & $5.9327 \mathrm{e}-4$ & $2.4243 \mathrm{e}-4$ & $9.8870 \mathrm{e}-5$ \\
BD-ADI [3] & & $2.2326 \mathrm{e}-3$ & $1.0149 \mathrm{e}-3$ & $4.4422 \mathrm{e}-4$ & $1.8953 \mathrm{e}-4$ \\
\hline
\end{tabular}

Example 5.3. Consider the following three-dimensional time-fractional subdiffusion equation

$$
\begin{cases}{ }_{C} D_{0, t}^{\beta} U=\left(\partial_{x}^{2}+\partial_{y}^{2}+\partial_{z}^{2}\right) U+f(x, y, z, t), & (x, y, z, t) \in \Omega \times(0,1], \\ U(x, y, z, 0)=\sin (\pi x) \sin (\pi y) \sin (\pi z), \quad(x, y, z) \in \bar{\Omega}, & \\ U(x, y, z, t)=0, \quad(x, y, z, t) \in \partial \Omega \times(0,1] & \end{cases}
$$

where $\Omega=(0,1) \times(0,1) \times(0,1)$. Choose a suitable right hand side function $f$ such that the exact solution to $(58)$ is

$$
U(x, y, t)=\left(t^{2+\beta}+t+1\right) \sin (\pi x) \sin (\pi y) \sin (\pi z) .
$$

Here, we test the space accuracy of the methods FDM II $(q)$ and CFDM II $(q)$, the $L^{2}$ errors at $t=1$ are shown in Table 7 . We observe that the methods FDM II $(q)$ are second-order accurate and CFDM II $(q)$ are fourth-order accurate in space. 


\begin{tabular}{c|ccr|crr}
\multicolumn{6}{c}{ Table 7: The $L^{2}$ errors at $t=1$ for Example $5.3, N=N_{1}=N_{2}=N_{3}, \beta=0.4, \tau=10^{-3}$} \\
\hline$N$ & FDM II (1) & order & cputime (s) & FDM II (2) & order & cputime (s) \\
\hline 10 & $8.4514 \mathrm{e}-3$ & & 10.7401 & $8.4514 \mathrm{e}-3$ & & 9.4637 \\
20 & $2.1055 \mathrm{e}-3$ & 2.01 & 62.3682 & $2.1055 \mathrm{e}-3$ & 2.01 & 53.4307 \\
30 & $9.3521 \mathrm{e}-4$ & 2.00 & 188.4161 & $9.3517 \mathrm{e}-4$ & 2.00 & 157.2630 \\
40 & $5.2595 \mathrm{e}-4$ & 2.00 & 651.8662 & $5.2592 \mathrm{e}-4$ & 2.00 & 517.7333 \\
\hline \hline$N$ & CFDM II (1) & order & cputime (s) & CFDM II (2) & order & cputime (s) \\
\hline 4 & $4.0268 \mathrm{e}-3$ & & 3.0570 & $4.0269 \mathrm{e}-3$ & & 2.4916 \\
8 & $2.4019 \mathrm{e}-4$ & 4.07 & 6.6952 & $2.4022 \mathrm{e}-4$ & 4.07 & 5.4640 \\
12 & $4.7003 \mathrm{e}-5$ & 4.02 & 16.0957 & $4.7038 \mathrm{e}-5$ & 4.02 & 13.3872 \\
16 & $1.4802 \mathrm{e}-5$ & 4.02 & 34.0454 & $1.4836 \mathrm{e}-5$ & 4.01 & 27.7393 \\
\hline
\end{tabular}

\section{Conclusion}

We have proposed two fully discrete ADI finite difference methods (FDMs) for the twodimensional time-fractional subdiffusion equation (1). The two ADI FDMs are unconditionally stable with convergence of order two in space and $(1+\beta)$ in time. In order to overcome the barrier on convergence order in time of the ADI methods, we propose two non-ADI FDMs for (1), where we employ the fast Fourier transform to solve the resulting linear system derived from these two non-ADI difference schemes. We present rigorous stability and convergence analysis and show that these two non-ADI methods are unconditionally stable and convergent of order two in both space and time. We also discuss how to improve the convergence rate in physical space using compact FDMs, and how to extend the methodology to three-dimensional time-fractional subdiffusion while we can still employ fast solvers with linearithmic complexity.

Compared with the direct solvers, the fast solvers presented here can reduce the computational cost from $O\left(N^{3}\right)$ (direct solver) to $O\left(N^{2} \log (N)\right)$ in space for two-dimensional problems, where $N$ is the grid points in each direction in space. Although the computational cost is a bit higher than that of the ADI FDMs $\left(O\left(N^{2}\right)\right)$ in physical space, numerical experiments show that non-ADI FDMs with fast solvers are competitive with the ADI algorithms.

The proposed methods can be readily extended to $d$-dimensional time-fractional anomalous diffusion and the computational cost in physical space is $O\left(N^{d} \log (N)\right)$. While the methods proposed here lead to faster algorithms, we still face the problem of storing the entire field at every time step, hence requiring a lot of memory, especially for long time integration. To this end, new methods like the ones proposed in [50] could reduce substantially the memory requirements for efficient long time integration.

\section{Appendix}

\section{A. Proofs}

Here we present the stability and error analysis for all the numerical methods in Sections 2 and 3 . We need the following lemmas for proofs. 
Lemma A.1 ([44]). Let $\left\{\omega_{k}\right\}$ be given by (8). Then we have

$$
\begin{aligned}
& \omega_{0}=1, \omega_{n}<0,\left|\omega_{n+1}\right|<\left|\omega_{n}\right|, \quad n=1,2, \ldots ; \\
& \omega_{0}=-\sum_{k=1}^{\infty} \omega_{k}>-\sum_{k=1}^{n} \omega_{k}>0, \quad n=1,2, \ldots ; \\
& b_{n-1}=\sum_{k=0}^{n-1} \omega_{k}=\frac{\Gamma(n-\beta)}{\Gamma(1-\beta) \Gamma(n)}=O\left(n^{-\beta}\right), \quad n \text { is sufficiently large. }
\end{aligned}
$$

Furthermore, $b_{n}-b_{n-1}=\omega_{n}<0$ for $n>0$, i.e., $b_{n}<b_{n-1}$.

Lemma A.2. Let $b_{n}$ be defined by (12). For any $\mathbf{G}=\left\{\mathbf{G}^{1}, \mathbf{G}^{2}, \mathbf{G}^{3}, \ldots\right\}$ and $\mathbf{q}$, where $\mathbf{G}^{j}, \mathbf{q} \in R^{\left(N_{1}+1\right) \times\left(N_{2}+1\right)}$, we have

$$
\begin{aligned}
A^{k}(\mathbf{G}, \mathbf{q}) & =\sum_{n=1}^{k}\left[b_{0}\left(\mathbf{G}^{n}, \mathbf{G}^{n}\right)-\sum_{j=1}^{n-1}\left(b_{n-j-1}-b_{n-j}\right)\left(\mathbf{G}^{j}, \mathbf{G}^{n}\right)-B_{n-1}\left(\mathbf{q}, \mathbf{G}^{n}\right)\right] \\
& \geq \frac{1}{2}\left[\sum_{n=1}^{k} b_{k-n}\left\|\mathbf{G}^{n}\right\|^{2}-C_{0}^{2}\|\mathbf{q}\|^{2} \sum_{n=1}^{k} b_{n-1}\right] \geq C k^{-\beta} \sum_{n=1}^{k}\left\|\mathbf{G}^{n}\right\|^{2}-C k^{1-\beta}\|\mathbf{q}\|^{2},
\end{aligned}
$$

where $C$ is a positive constant dependent only on $\beta$ and $C_{0}$, and $C_{0}$ satisfies $\left|B_{n-1}\right| \leq C_{0} b_{n-1}$.

The proof of Lemma A.2 is similar to that of Lemma 3.7 in [44], which is omitted here.

Remark A.1. If the coefficients $\left(b_{n-j-1}-b_{n-j}\right)$ in (A.2) are replaced by $(-1)^{\sigma(j)}\left(b_{n-j-1}-\right.$ $\left.b_{n-j}\right)$, where $\sigma(j)$ is chosen randomly as 0 or 1 , then (A.2) still holds.

Lemma A.3. $(\cdot, \cdot)_{\mathcal{A}}$ defined by $(37)$ and $(\cdot, \cdot)_{\mathcal{H}}$ defined by $(38)$ are inner products.

Proof. Let $\mathbf{u}, \mathbf{v} \in \mathcal{V}_{0}$. Then we can easily verify that $(\mathbf{u}, \mathbf{v})_{\mathcal{A}}=(\mathbf{v}, \mathbf{u})_{\mathcal{A}}$ by the property

$$
\left(\delta_{x}^{2} \mathbf{u}, \mathbf{v}\right)=-\left(\delta_{x} \mathbf{u}, \delta_{x} \mathbf{v}\right)=\left(\mathbf{u}, \delta_{x}^{2} \mathbf{v}\right), \quad\left(\delta_{y}^{2} \mathbf{u}, \mathbf{v}\right)=-\left(\delta_{y} \mathbf{u}, \delta_{y} \mathbf{v}\right)=\left(\mathbf{u}, \delta_{y}^{2} \mathbf{v}\right) .
$$

The bilinear of $(\mathbf{u}, \mathbf{v})_{\mathcal{A}}$ is obvious. Next we need only to prove that $(\cdot, \cdot)_{\mathcal{A}}$ is positive definite. It is very easy to get that

$$
(\mathbf{u}, \mathbf{v})_{\mathcal{A}}=(\operatorname{vec}(\underline{\mathbf{u}}))^{T} \mathcal{M} \operatorname{vec}(\underline{\mathbf{v}}),
$$

where $\mathcal{M}=E_{N_{2}-1} \otimes E_{N_{1}-1}-\frac{1}{12}\left(E_{N_{2}-1} \otimes S_{N_{1}-1}+S_{N_{2}-1} \otimes E_{N_{1}-1}\right), \underline{\mathbf{u}}, \underline{\mathbf{v}}$ are defined as in (25), and $\operatorname{vec}(\underline{\mathbf{u}})$ creates a column vector from the matrix $\underline{\mathbf{u}}$, i.e.,

$$
\operatorname{vec}(\underline{\mathbf{u}})=\left(u_{1,1}, u_{2,1}, \ldots, u_{N_{1}-1,1}, u_{1,2}, u_{2,2}, \ldots, u_{N_{1}-1,2}, \ldots, u_{1, N_{2}-1}, u_{2, N_{2}-1}, \ldots, u_{N_{1}-1, N_{2}-1}\right)^{T} .
$$

It is easy to know that the eigenvalues of $\mathcal{M}$ are $1-\frac{1}{12}\left(\lambda_{i}^{(1)}+\lambda_{j}^{(2)}\right) \geq 1-\frac{8}{12}=\frac{1}{3}$, where $\lambda_{j}^{(k)}$ is defined in $(27)$. Hence, $(\cdot, \cdot)_{\mathcal{A}}$ is positive definite. Therefore, $(\cdot, \cdot)_{\mathcal{A}}$ defines an inner product. We can similarly prove that $(\cdot, \cdot)_{\mathcal{H}}$ defines an inner product, which ends the proof. 
In order to prove the stability and convergence, we need the following inequalities [44]

$$
\left|B_{n}^{(q)}\right| \leq C n^{-1}, \quad\left|C_{n}^{(q)}\right| \leq C n^{-1}, \quad q=1,2, \quad n>0,
$$

where $C$ is a positive constant independent of $n$ and $\tau$.

Next, we present only the detailed proofs for Theorems 3.3, 3.4, and 2.1. The stability and convergence analysis for other theorems are very similar, which is omitted here.

\section{A.1. Proof of Theorem 3.3}

Proof. We just prove (39) for $q=1$, which is the same for $q=2$. From (34), one easily has

$$
\left(D^{(n)} \mathbf{u}, \mathbf{u}^{n}\right)_{\mathcal{A}}=-\mu\left(L_{1}^{(n)} \mathbf{u}, \mathbf{u}^{n}\right)_{\mathcal{H}}-\mu B_{n}^{(1)}\left(\mathbf{u}^{0}, \mathbf{u}^{n}\right)_{\mathcal{H}}-\mu C_{n}^{(1)}\left(\mathbf{u}^{1}-\mathbf{u}^{0}, \mathbf{u}^{n}\right)_{\mathcal{H}}+\frac{1}{\tau^{\beta}}\left(\mathbf{F}^{n}, \mathbf{u}^{n}\right)_{\mathcal{A}},
$$

where $\left(\mathbf{F}^{n}\right)_{i, j}=F_{i, j}^{n}=\sum_{k=0}^{n} \omega_{n-k}\left[D_{0, t}^{-\beta} f\left(x_{i}, y_{j}, t\right)\right]_{t=t_{k}}\left(0 \leq i \leq N_{1}, 0 \leq j \leq N_{2}\right)$. Using the property $b_{n}-b_{n-1}=\omega_{n}$ (see Lemma A.1), we rewrite (A.4) as

$$
\begin{aligned}
\left\|\mathbf{u}^{n}\right\| \|_{3}^{2}= & \left(\mathbf{u}^{n}, \mathbf{u}^{n}\right)_{\mathcal{A}}+\mu(\tau / 2)^{\beta}\left(\mathbf{u}^{n}, \mathbf{u}^{n}\right)_{\mathcal{H}} \\
= & \sum_{k=1}^{n}\left(b_{k-1}-b_{k}\right)\left[\left(\mathbf{u}^{n-k}, \mathbf{u}^{n}\right)_{\mathcal{A}}+\mu(\tau / 2)^{\beta}(-1)^{k}\left(\mathbf{u}^{n-k}, \mathbf{u}^{n}\right)_{\mathcal{H}}\right] \\
& +b_{n}\left(\mathbf{u}^{0}, \mathbf{u}^{n}\right)_{\mathcal{A}}-\mu \tau^{\beta} B_{n}^{(1)}\left(\mathbf{u}^{0}, \mathbf{u}^{n}\right)_{\mathcal{H}}-\mu \tau^{\beta} C_{n}^{(1)}\left(\mathbf{u}^{1}-\mathbf{u}^{0}, \mathbf{u}^{n}\right)_{\mathcal{H}}+\left(\mathbf{F}^{n}, \mathbf{u}^{n}\right)_{\mathcal{A}} .
\end{aligned}
$$

Applying Lemma A.3, using (A.5), $b_{n}-b_{n-1} \leq 0$, and the Cauchy-Schwartz inequality, we have

$$
\begin{aligned}
\left\|\mathbf{u}^{n} \mid\right\|_{3}^{2} \leq & \frac{1}{2} \sum_{k=1}^{n}\left(b_{k-1}-b_{k}\right)\left[\left\|\mathbf{u}^{n-k}\right\|_{\mathcal{A}}^{2}+\left\|\mathbf{u}^{n}\right\|_{\mathcal{A}}^{2}+\mu(\tau / 2)^{\beta}\left(\left\|\mathbf{u}^{n-k}\right\|_{\mathcal{H}}^{2}+\left\|\mathbf{u}^{n}\right\|_{\mathcal{H}}^{2}\right)\right] \\
& +b_{n}\left\|\mathbf{u}^{0}\right\|_{\mathcal{A}}^{2}+\frac{b_{n}}{4}\left\|\mathbf{u}^{n}\right\|_{\mathcal{A}}^{2}+\frac{1}{b_{n}}\left\|\mathbf{F}^{n}\right\|_{\mathcal{A}}^{2}+\frac{b_{n}}{4}\left\|\mathbf{u}^{n}\right\|_{\mathcal{A}}^{2} \\
& +\mu\left|B_{n}^{(1)}\right| \tau^{\beta}\left(\epsilon_{1}\left\|\mathbf{u}^{n}\right\|_{\mathcal{H}}^{2}+\frac{1}{4 \epsilon_{1}}\left\|\mathbf{u}^{0}\right\|_{\mathcal{H}}^{2}\right)+\mu\left|C_{n}^{(1)}\right| \tau^{\beta}\left(\epsilon_{2}\left\|\mathbf{u}^{n}\right\|_{\mathcal{H}}^{2}+\frac{1}{4 \epsilon_{2}}\left\|\mathbf{u}^{1}-\mathbf{u}^{0}\right\|_{\mathcal{H}}^{2}\right) \\
= & \frac{1}{2}\left|\left\|\mathbf{u}^{n}\left|\left\|_{3}^{2}+\frac{1}{2} \sum_{k=1}^{n}\left(b_{k-1}-b_{k}\right)\right\| \mathbf{u}^{n-k}\right|\right\|_{3}^{2}+\frac{1}{b_{n}}\left\|\mathbf{F}^{n}\right\|_{\mathcal{A}}^{2}+b_{n}\left\|\mathbf{u}^{0}\right\|_{\mathcal{A}}^{2}\right. \\
& +\left(-\frac{1}{2} b_{n} \mu(\tau / 2)^{\beta}+\epsilon_{1} \mu\left|B_{n}^{(1)}\right| \tau^{\beta}+\epsilon_{2} \mu\left|C_{n}^{(1)}\right| \tau^{\beta}\right)\left\|\mathbf{u}^{n}\right\|_{\mathcal{H}}^{2} \\
& +\frac{\mu\left|B_{n}^{(1)}\right| \tau^{\beta}}{4 \epsilon_{1}}\left\|\mathbf{u}^{0}\right\|_{\mathcal{H}}^{2}+\frac{\mu\left|C_{n}^{(1)}\right| \tau^{\beta}}{4 \epsilon_{2}}\left\|\mathbf{u}^{1}-\mathbf{u}^{0}\right\|_{\mathcal{H}}^{2},
\end{aligned}
$$

where $\epsilon_{1}$ and $\epsilon_{2}$ are suitable positive constants independent of $n, \tau, \Delta x, \Delta y$, and $T$ satisfying

$$
-\frac{1}{2} b_{n}(1 / 2)^{\beta}+\epsilon_{1}\left|B_{n}^{(1)}\right|+\epsilon_{2}\left|C_{n}^{(1)}\right| \leq 0,
$$


which can be deduced from Lemma A.1 and (A.3). From Lemma A.1, we have $1 / b_{n} \leq C_{\beta} n^{\beta}$, $C_{\beta}$ is only dependent on $\beta$. Hence, we have from (A.6)

$$
\left\|\left|\mathbf { u } ^ { n } \left\|\left.\right|_{3} ^{2} \leq \sum_{k=1}^{n}\left(b_{k-1}-b_{k}\right)\left|\left\|\mathbf{u}^{n-k} \mid\right\|_{3}^{2}+\frac{2}{b_{n}}\left\|\mathbf{F}^{n}\right\|_{\mathcal{A}}^{2}+2 b_{n}\left\|\mathbf{u}^{0}\right\|_{\mathcal{A}}^{2}+C \tau^{\beta} b_{n}\left(\left\|\mathbf{u}^{0}\right\|_{\mathcal{H}}^{2}+\left\|\mathbf{u}^{1}\right\|_{\mathcal{H}}^{2}\right),\right.\right.\right.\right.
$$

where $C$ is a positive constant independent of $n, \Delta x, \Delta y, \tau$, and $T$. Noticing that

$$
\frac{\tau^{2 \beta}}{b_{n}}=b_{n} \frac{1}{b_{n}^{2}} \frac{T^{2 \beta}}{n_{T}^{2 \beta}} \leq b_{n} C_{\beta}^{2} T^{2 \beta}\left(\frac{n}{n_{T}}\right)^{2 \beta} \leq\left(C_{\beta} T^{\beta}\right)^{2} b_{n}
$$

we have

$$
\frac{2}{b_{n}}\left\|\mathbf{F}^{n}\right\|_{\mathcal{A}}^{2} \leq \frac{\widetilde{C}_{3} \tau^{2 \beta}}{b_{n}} \max _{0 \leq t \leq t_{n}}\|\mathbf{f}(t)\|_{\mathcal{A}}^{2} \leq C_{3} b_{n} \max _{0 \leq t \leq t_{n}}\|\mathbf{f}(t)\|_{\mathcal{A}}^{2} \leq \frac{5}{3} C_{3} b_{n} \max _{0 \leq t \leq t_{n}}\|\mathbf{f}(t)\|^{2} .
$$

Letting $n=1$ in (A.5), we can similarly obtain

$$
\left\|\left|\mathbf{u}^{1}\right|\right\|_{3}^{2} \leq C\left|\left\|\mathbf{u}^{0}\right\|\right|_{3}^{2}+\tilde{C}\left(\left\|\mathbf{f}^{0}\right\|^{2}+\left\|\mathbf{f}^{1}\right\|^{2}\right)
$$

where $\tilde{C}>0$ is independent of $n, \tau, \Delta x, \Delta y$, and $T$. Combining (A.7)-(A.9) yields

$$
\left\|\left|\mathbf{u}^{n}\left\|\left.\right|_{3} ^{2} \leq \sum_{k=1}^{n}\left(b_{k-1}-b_{k}\right)\right\|\left\|\mathbf{u}^{n-k}\right\|\right|_{3}^{2}+C_{1} b_{n}\right\|\left|\mathbf{u}^{0}\left\|\left.\right|_{3} ^{2}+C_{2} b_{n} \max _{0 \leq t \leq t_{n}}\right\| \mathbf{f}(t) \|^{2}\right.
$$

where $C_{1}$ and $C_{2}$ are positive constants independent of $n, \Delta x, \Delta y, \tau$, and $C_{1}$ is also independent of $T$. Denote by

$$
E=C_{1}\left\|\left|\mathbf{u}^{0}\left\|\left.\right|_{3} ^{2}+C_{2} \max _{0 \leq t \leq T}\right\| \mathbf{f}(t) \|^{2}\right.\right.
$$

Then we have from (A.10)

$$
\left.\left\|\mathbf{u}^{n}\right\|\right|_{3} ^{2} \leq \sum_{k=1}^{n}\left(b_{k-1}-b_{k}\right)\left\|\mid \mathbf{u}^{n-k}\right\|_{3}^{2}+b_{n} E .
$$

From here and by the induction method, we reach the conclusion (39). The proof is completed.

\section{A.2. Proof of Theorem 3.4}

Proof. Denoting $\left(\mathbf{e}^{n}\right)_{i, j}=e_{i, j}^{n}=U\left(x_{i}, y_{j}, t_{n}\right)-u_{i, j}^{n}$, we obtain the error equation for (34) as

$$
D^{(n)} \mathcal{A} e_{i, j}=\mathcal{H}\left(\mu L_{1}^{(n)} e_{i, j}+\mu B_{n}^{(1)} e_{i, j}^{0}+\mu C_{n}^{(1)}\left(e_{i, j}^{1}-e_{i, j}^{0}\right)\right)+R_{i, j}^{n},
$$

where

$$
R_{i, j}^{n}=O\left(\tau^{2}+\Delta x^{4}+\Delta y^{4}+\Delta x^{2} \Delta y^{2}\right)
$$

According to Theorem 3.3, we have

$$
\left\|\mathbf{e}^{n}\right\| \leq\left.\left\|\mathbf{e}^{n}\right\|\right|_{q} \leq\left. C_{1}\left\|\mathbf{e}^{0}\right\|\right|_{q} ^{2}+C_{2} \max _{0 \leq k \leq n_{T}}\left\|\mathbf{R}^{k}\right\|^{2}, \quad q=3,4 .
$$

With the fact that $\left\|\left|\mathbf{e}^{0} \|\right|_{q}=0\right.$ and the estimate (A.11), we obtain (40). 
The proofs of stability and convergence for difference schemes (24), (42), and (54) are almost the same to that of (34), which is omitted here.

\section{A.3. Proof of Theorem 2.1}

Let us define $\mathcal{B} u_{i, j}=\delta_{x}^{2} \delta_{y}^{2} u_{i, j}$ for $1 \leq i \leq N_{1}-1,1 \leq j \leq N_{2}-1$. Then $(\cdot, \cdot)_{\mathcal{B}}$ defined by

$$
(\mathbf{u}, \mathbf{v})_{\mathcal{B}}=\Delta x \Delta y \sum_{i=1}^{N_{1}-1} \sum_{j=1}^{N_{2}-1}\left(\mathcal{B} u_{i, j}\right) v_{i, j}
$$

is an inner product with norm $\|\cdot\|_{\mathcal{B}}=\sqrt{(\cdot, \cdot)_{\mathcal{B}}}$, where $\mathbf{u}, \mathbf{v} \in \mathbb{R}^{\left(N_{1}+1\right) \times\left(N_{2}+1\right)}$ with $(\mathbf{u})_{i, j}=$ $u_{i, j},(\mathbf{v})_{i, j}=v_{i, j}\left(0 \leq i \leq N_{1}, 0 \leq j \leq N_{2}\right)$. We can also define the inner product $(\cdot, \cdot)_{\mathcal{C}}$ and the norm $\|\cdot\|_{\mathcal{C}}$ as

$$
(\mathbf{u}, \mathbf{v})_{\mathcal{C}}=-\Delta x \Delta y \sum_{i=1}^{N_{1}-1} \sum_{j=1}^{N_{2}-1}\left(\mathcal{C} u_{i, j}\right) v_{i, j}, \quad\|\mathbf{u}\|_{\mathcal{C}}=\sqrt{(\mathbf{u}, \mathbf{u})_{\mathcal{C}}}
$$

where $\mathcal{C} u_{i, j}=\left(\delta_{x}^{2}+\delta_{y}^{2}\right) u_{i, j}\left(1 \leq i \leq N_{1}-1,1 \leq j \leq N_{2}-1\right)$.

The inner products $(\cdot, \cdot)_{\mathcal{B}}$ and $(\cdot, \cdot)_{\mathcal{C}}$ can be similarly proved as that of Lemma A.3.

Proof. We consider only the case $q=1$. By (17) and $\omega_{k}=b_{k}-b_{k-1}$, we have

$$
\begin{aligned}
& \frac{1}{\tau^{\beta}}\left[b_{0}\left(\mathbf{u}^{n}, \mathbf{u}^{n}\right)-\sum_{k=1}^{n-1}\left(b_{k-1}-b_{k}\right)\left(\mathbf{u}^{n-k}, \mathbf{u}^{n}\right)-b_{n-1}\left(\mathbf{u}^{0}, \mathbf{u}^{n}\right)\right] \\
& +\mu^{2} \tau^{\beta}\left(2^{-\beta}+\delta_{n, 1} C_{1}^{(1)}\right)^{2}\left(\mathbf{u}^{n}-\mathbf{u}^{n-1}, \mathbf{u}^{n}\right)_{\mathcal{B}} \\
= & -\frac{\mu}{2^{\beta}}\left[b_{0}\left(\mathbf{u}^{n}, \mathbf{u}^{n}\right)_{\mathcal{C}}-\sum_{k=1}^{n-1}(-1)^{n-k}\left(b_{k-1}-b_{k}\right)\left(\mathbf{u}^{n-k}, \mathbf{u}^{n}\right)_{\mathcal{C}}-(-1)^{n}\left(b_{n-1}-b_{n}\right)\left(\mathbf{u}^{0}, \mathbf{u}^{n}\right)_{\mathcal{C}}\right] \\
& +\left(\mathbf{F}^{n}, \mathbf{u}^{n}\right) .
\end{aligned}
$$

Applying Lemma A.2, Remark A.1, and the Cauchy-Schwartz inequality, we obtain

$$
\begin{aligned}
& \frac{1}{2} \frac{1}{\tau^{\beta}}\left(\sum_{n=1}^{K} b_{K-n}\left\|\mathbf{u}^{n}\right\|^{2}-\left\|\mathbf{u}^{0}\right\|^{2} \sum_{n=1}^{K} b_{n-1}\right)+\mu^{2} \tau^{\beta} \sum_{n=1}^{K}\left(2^{-\beta}+\delta_{n, 1} C_{n}^{(1)}\right)^{2}\left\|\mathbf{u}^{n}\right\|_{\mathcal{B}}^{2} \\
& +\frac{1}{2} \frac{\mu}{2^{\beta}}\left(\sum_{n=1}^{K} b_{K-n}\left\|\mathbf{u}^{n}\right\|_{\mathcal{C}}^{2}-\left\|\mathbf{u}^{0}\right\|_{\mathcal{C}}^{2} \sum_{n=1}^{K} b_{n-1}\right) \\
\leq & \sum_{n=1}^{K}\left(\mathbf{F}^{n}, \mathbf{u}^{n}\right)+\mu^{2} \tau^{\beta} \sum_{n=1}^{K}\left(2^{-\beta}+\delta_{n, 1} C_{n}^{(1)}\right)^{2}\left\|\mathbf{u}^{n-1}\right\|_{\mathcal{B}}^{2} \\
\leq & \sum_{n=1}^{K}\left(\frac{1}{4} \frac{b_{K-n}}{\tau^{\beta}}\left\|\mathbf{u}^{n}\right\|^{2}+\frac{\tau^{\beta}}{b_{K-n}}\left\|\mathbf{F}^{n}\right\|^{2}\right)+\mu^{2} \tau^{\beta} \sum_{n=1}^{K}\left(2^{-\beta}+\delta_{n, 1} C_{n}^{(1)}\right)^{2}\left\|\mathbf{u}^{n-1}\right\|_{\mathcal{B}}^{2} .
\end{aligned}
$$


From Lemma A.1, we has $\tau^{\beta} \leq C b_{n}$. Hence, we have from the above equation

$$
\begin{aligned}
& \sum_{n=1}^{K} b_{K-n}\left(\left\|\mathbf{u}^{n}\right\|^{2}+\tau^{\beta} 2^{1-\beta} \mu\left\|\mathbf{u}^{n}\right\|_{\mathcal{C}}^{2}\right)+4 \mu^{2} \tau^{2 \beta}\left[2^{-2 \beta}\left\|\mathbf{u}^{K}\right\|_{\mathcal{B}}^{2}+\left(\left(2^{-\beta}+C_{1}^{(1)}\right)^{2}-2^{-2 \beta}\right)\left\|\mathbf{u}^{1}\right\|_{\mathcal{B}}^{2}\right] \\
\leq & 4 \tau^{\beta} \sum_{n=1}^{K} \frac{\tau^{\beta}}{b_{k-n}}\left\|\mathbf{F}^{n}\right\|^{2}+4\left(\left\|\mathbf{u}^{0}\right\|^{2}+\tau^{\beta} 2^{1-\beta} \mu\left\|\mathbf{u}^{0}\right\|_{\mathcal{C}}^{2}\right) \sum_{n=1}^{K} b_{n-1}+4 \mu^{2} \tau^{2 \beta}\left(2^{-\beta}+C_{1}^{(1)}\right)^{2}\left\|\mathbf{u}^{0}\right\|_{\mathcal{B}}^{2} \\
\leq & C \tau^{\beta} \sum_{n=1}^{K}\left\|\mathbf{F}^{n}\right\|^{2}+4\left(\left\|\mathbf{u}^{0}\right\|^{2}+\tau^{\beta} 2^{1-\beta} \mu\left\|\mathbf{u}^{0}\right\|_{\mathcal{C}}^{2}\right) \sum_{n=1}^{K} b_{n-1}+4 \mu^{2} \tau^{2 \beta}\left(2^{-\beta}+C_{1}^{(1)}\right)^{2}\left\|\mathbf{u}^{0}\right\|_{\mathcal{B}}^{2} .
\end{aligned}
$$

With $\tau^{\beta} \leq C b_{n}$ again, the property $\sum_{n=1}^{K} b_{n-1}=O\left(K^{1-\beta}\right)$, and (A.16) lead to

$$
\begin{aligned}
& \tau \sum_{n=1}^{K}\left\|\mathbf{u}^{n}\right\|^{2} \leq \tau \sum_{n=1}^{K}\left(\left\|\mathbf{u}^{n}\right\|^{2}+\tau^{\beta} 2^{1-\beta} \mu\left\|\mathbf{u}^{n}\right\|_{\mathcal{C}}^{2}\right) \\
\leq & C \tau \sum_{n=1}^{K}\left\|\mathbf{F}^{n}\right\|^{2}+C\left(\left\|\mathbf{u}^{0}\right\|^{2}+\tau^{\beta} 2^{1-\beta} \mu\left\|\mathbf{u}^{0}\right\|_{\mathcal{C}}^{2}\right)+4 \mu^{2} \tau^{1+\beta}\left(2^{-\beta}+C_{1}^{(1)}\right)^{2}\left\|\mathbf{u}^{0}\right\|_{\mathcal{B}}^{2} .
\end{aligned}
$$

Note that $\sum_{k=0}^{n}\left|\omega_{k}\right|=\omega_{0}+\sum_{k=1}^{n}\left|\omega_{k}\right|<2 \omega_{0}=2$. We have

$$
\left\|\mathbf{F}^{n}\right\|=\left\|\sum_{k=0}^{n}(-1)^{k} \omega_{k} \mathbf{f}^{n-k}\right\| \leq \sum_{k=0}^{n}\left|\omega_{k}\right| \max _{0 \leq k \leq n_{T}}\left\|\mathbf{f}^{k}\right\| \leq C_{1} \max _{0 \leq k \leq n_{T}}\left\|\mathbf{f}^{k}\right\| .
$$

Combining (A.17) and (A.18), and using $\left\|\mathbf{u}^{n}\right\|_{\mathcal{B}}=\left\|\delta_{x} \delta_{y} \mathbf{u}^{n}\right\|$ and $\left\|\delta_{x} \mathbf{u}^{n}\right\|^{2}+\left\|\delta_{y} \mathbf{u}^{n}\right\|^{2}=\left\|\mathbf{u}^{n}\right\|_{\mathcal{C}}^{2}$ yields (22). The proof is completed.

From Theorem 2.1, we can readily obtain Theorem 2.2, which is omitted.

\section{References}

[1] R. Metzler, J. Klafter, The random walk's guide to anomalous diffusion: a fractional dynamics approach, Phys. Rep. 339 (1) (2000) 1-77.

[2] M. Cui, Convergence analysis of high-order compact alternating direction implicit schemes for the two-dimensional time fractional diffusion equation, Numer. Algorithms 62 (3) (2013) 383-409.

[3] Y.-N. Zhang, Z.-Z. Sun, Alternating direction implicit schemes for the two-dimensional fractional subdiffusion equation, J. Comput. Phys. 230 (24) (2011) 8713-8728.

[4] I. Podlubny, Fractional differential equations, Academic Press, Inc., San Diego, CA, 1999.

[5] M. Chen, W. Deng, A second-order numerical method for two-dimensional two-sided space fractional convection diffusion equation, Appl. Math. Model. 38 (13) (2014) 3244-3259.

[6] F. Liu, P. Zhuang, I. Turner, V. Anh, K. Burrage, A semi-alternating direction method for a 2-D fractional Fitzhugh-Nagumo monodomain model on an approximate irregular domain, J. Comput. Phys. 293 (2015) 252-263.

[7] M. M. Meerschaert, H.-P. Scheffler, C. Tadjeran, Finite difference methods for two-dimensional fractional dispersion equation, J. Comput. Phys. 211 (1) (2006) 249-261.

[8] C. Tadjeran, M. M. Meerschaert, A second-order accurate numerical method for the two-dimensional fractional diffusion equation, J. Comput. Phys. 220 (2) (2007) 813-823. 
[9] H. Wang, N. Du, Fast alternating-direction finite difference methods for three-dimensional spacefractional diffusion equations, J. Comput. Phys. 258 (2014) 305-318.

[10] W. Bu, Y. Tang, J. Yang, Galerkin finite element method for two-dimensional Riesz space fractional diffusion equations, J. Comput. Phys. 276 (2014) 26-38.

[11] C.-M. Chen, F. Liu, A numerical approximation method for solving a three-dimensional space Galilei invariant fractional advection-diffusion equation, J. Appl. Math. Comput. 30 (1-2) (2009) 219-236.

[12] L. Li, D. Xu, Alternating direction implicit-Euler method for the two-dimensional fractional evolution equation, J. Comput. Phys. 236 (2013) 157-168.

[13] S. Vong, Z. Wang, A compact difference scheme for a two dimensional fractional Klein-Gordon equation with Neumann boundary conditions, J. Comput. Phys. 274 (2014) 268-282.

[14] X. Zhao, Z.-Z. Sun, Compact Crank-Nicolson schemes for a class of fractional Cattaneo equation in inhomogeneous medium, J. Sci. Comput. 62 (2015) 747-771.

[15] S. Zhai, X. Feng, Y. He, An unconditionally stable compact ADI method for three-dimensional timefractional convection-diffusion equation, J. Comput. Phys. 269 (2014) 138-155.

[16] N. Zhang, W. Deng, Y. Wu, Finite difference/element method for a two-dimensional modified fractional diffusion equation, Adv. Appl. Math. Mech. 4 (4) (2012) 496-518.

[17] X. Zhang, P. Huang, X. Feng, L. Wei, Finite element method for two-dimensional time-fractional Tricomi-type equations, Numer. Methods Partial Differential Equations 29 (4) (2013) 1081-1096.

[18] Y.-N. Zhang, Z.-Z. Sun, X. Zhao, Compact alternating direction implicit scheme for the two-dimensional fractional diffusion-wave equation, SIAM J. Numer. Anal. 50 (3) (2012) 1535-1555.

[19] M. Abbaszadeh, A. Mohebbi, A fourth-order compact solution of the two-dimensional modified anomalous fractional sub-diffusion equation with a nonlinear source term, Comput. Math. Appl. 66 (8) (2013) $1345-1359$.

[20] C.-M. Chen, F. Liu, V. Anh, Numerical analysis of the Rayleigh-Stokes problem for a heated generalized second grade fluid with fractional derivatives, Appl. Math. Comput. 204 (1) (2008) 340-351.

[21] F. Zeng, C. Li, F. Liu, K. Burrage, I. Turner, V. Anh, A Crank-Nicolson ADI spectral method for a two-dimensional Riesz space fractional nonlinear reaction-diffusion equation, SIAM J. Numer. Anal. 52 (2014) 2599-2622.

[22] C.-C. Ji, Z-Z. Sun, A high-order compact finite difference scheme for the fractional sub-diffusion equation, J. Sci. Comput. 64 (2015) 959-985.

[23] C.-C. Ji, Z-Z. Sun, The high-order compact numerical algorithms for the two-dimensional fractional sub-diffusion equation, Appl. Math. Comput. 269 (2015) 775-791.

[24] Z. Wang, S. Vong, Compact difference schemes for the modified anomalous fractional sub-diusion equation and the fractional diffusion-wave equation, J. Comput. Phys. 277 (2014) 1-15.

[25] W. Bu, Y. Tang, J. Yang, Finite difference/finite element method for two-dimensional space and time fractional Bloch-Torrey equations, J. Comput. Phys. 293(2015) 264-279.

[26] M. Chen, W. Deng, Y. Wu, Superlinearly convergent algorithms for the two-dimensional space-time Caputo-Riesz fractional diffusion equation, Appl. Numer. Math. 70 (2013) 22-41.

[27] S. Saha Ray, A. K. Gupta, A two-dimensional Haar wavelet approach for the numerical simulations of time and space fractional Fokker-Planck equations in modelling of anomalous diffusion systems, J. Math. Chem. 52 (8) (2014) 2277-2293.

[28] Q. Yu, F. Liu, I. Turner, K. Burrage, A computationally effective alternating direction method for the space and time fractional Bloch-Torrey equation in 3-D, Appl. Math. Comput. 219 (8) (2012) 4082-4095.

[29] P. Zhuang, F. Liu, Implicit difference approximation for the two-dimensional space-time fractional diffusion equation, J. Appl. Math. Comput. 25 (1-2) (2007) 269-282.

[30] C. Li, F. Zeng, Numerical Methods for Fractional Calculus, CRC Press, 2015.

[31] S. Chen, F. Liu, ADI-Euler and extrapolation methods for the two-dimensional fractional advectiondispersion equation, J. Appl. Math. Comput. 26 (1-2) (2008) 295-311.

[32] S. Chen, F. Liu, K. Burrage, Numerical simulation of a new two-dimensional variable-order fractional percolation equation in non-homogeneous porous media, Comput. Math. Appl. 67 (9) (2014) 1673-1681. 
[33] F. Liu, S. Chen, I. Turner, K. Burrage, V. Anh, Numerical simulation for two-dimensional riesz space fractional diffusion equations with a nonlinear reaction term, Cent. Eur. J. Phys. 11 (10) (2013) 12211232.

[34] Q. Liu, F. Liu, I. Turner, V. Anh, Numerical simulation for the 3D seepage flow with fractional derivatives in porous media, IMA J. Appl. Math. 74 (2) (2009) 201-229.

[35] Z. Wang, S. Vong, A high-order exponential ADI scheme for two dimensional time fractional convectiondiffusion equations, Comput. Math. Appl. 68 (3) (2014) 185-196.

[36] J. Chen, F. Liu, Q. Liu, X. Chen, V. Anh, I. Turner, K. Burrage, Numerical simulation for the threedimension fractional sub-diffusion equation, Appl. Math. Model. 38 (15-16) (2014) 3695-3705.

[37] M. Cui, Compact alternating direction implicit method for two-dimensional time fractional diffusion equation, J. Comput. Phys. 231 (6) (2012) 2621-2633.

[38] Y.-N. Zhang, Z.-Z. Sun, Error analysis of a compact ADI scheme for the 2D fractional subdiffusion equation, J. Sci. Comput. 59 (1) (2014) 104-128.

[39] J. Douglas, Jr., H. H. Rachford, Jr., On the numerical solution of heat conduction problems in two and three space variables, Trans. Amer. Math. Soc. 82 (1956) 421-439.

[40] J. Douglas, Jr., S. Kim, Improved accuracy for locally one-dimensional methods for parabolic equations, Math. Models Methods Appl. Sci. 11 (9) (2001) 1563-1579.

[41] D. W. Peaceman, H. H. Rachford, Jr., The numerical solution of parabolic and elliptic differential equations, J. Soc. Indust. Appl. Math. 3 (1955) 28-41.

[42] C.-M. Chen, F. Liu, I. Turner, V. Anh, Numerical schemes and multivariate extrapolation of a twodimensional anomalous sub-diffusion equation, Numer. Algorithms 54 (1) (2010) 1-21.

[43] K. Diethelm, N. J. Ford, A. D. Freed, Detailed error analysis for a fractional Adams method, Numer. Algorithms 36 (1) (2004) 31-52.

[44] F. Zeng, C. Li, F. Liu, I. Turner, Numerical algorithms for time-fractional subdiffusion equation with second-order accuracy, SIAM J. Sci. Comput. 37 (2015) A55-A78.

[45] F. Zeng, Second-order stable finite difference schemes for the time-fractional diffusion-wave equation, J. Sci. Comput. 65 (2014) 411-430.

[46] H. Wang, T. S. Basu, A fast finite difference method for two-dimensional space-fractional diffusion equations, SIAM J. Sci. Comput. 34 (5) (2012) A2444-A2458.

[47] C. Lubich, Discretized fractional calculus, SIAM J. Math. Anal. 17 (3) (1986) 704-719.

[48] B. L. Buzbee, G. H. Golub, C. W. Nielson, On direct methods for solving Poisson's equations, SIAM J. Numer. Anal. 7 (1970) 627-656.

[49] D. Kulkarni, D. Schmidt, S.-K. Tsui, Eigenvalues of tridiagonal pseudo-Toeplitz matrices, Linear Algebra Appl. 297 (1-3) (1999) 63-80.

[50] C. Lubich, A. Schädle, Fast convolution for nonreflecting boundary conditions, SIAM J. Sci. Comput. 24 (2002) 161-182.

\section{Acknowledgements}

This work was supported by the MURI/ARO on "Fractional PDEs for Conservation Laws and Beyond: Theory, Numerics and Applications (W911NF-15-1-0562)", and also by NSF (DMS 1216437). The second author of this work was partially supported by a startup fund from WPI. 